\title{
Wind turbines using self-excited three-phase induction generators: an innovative solution for voltage-frequency control
}

\author{
J.F. Brudny, R. Pusca ${ }^{a}$, and H. Roisse \\ Laboratoire Systèmes Électrotechniques et Environnement, Université d'Artois, Faculté des Sciences Appliquées, Technoparc \\ Futura, 62400 Béthune, France
}

Received: 23 July 2007 / Received in final form: 4 April 2008 / Accepted: 9 April 2008

Published online: 19 July 2008 - (C) EDP Sciences

\begin{abstract}
A considerable number of communities throughout the world, most of them isolated, need hybrid energy solutions either for rural electrification or for the reduction of diesel use. Despite several research projects and demonstrations which have been conducted in recent years, wind-diesel technology remains complex and much too costly. Induction generators are the most robust and common for wind energy systems but this option is a serious challenge for electrical regulation. When a wind turbine is used in an off-grid configuration, either continuously or intermittently, precise and robust regulation is difficult to attain. The voltage parameter regulation option, as was experienced at several remote sites (on islands and in the arctic for example), is a safe, reliable and relatively simple technology, but does not optimize the wave quality and creates instabilities. These difficulties are due to the fact that no theory is available to describe the system, due to the inverse nature of the problem. In order to address and solve the problem of the unstable operation of this wind turbine generator, an innovative approach is described, based on a different induction generator single phase equivalent circuit.
\end{abstract}

PACS. 84.50.+d Electric motors - 84.60.-h Direct energy conversion and storage - 89.30.Ee Hydroelectric, hydrothermal, geothermal and wind power - 89.30.-g Energy resources

\section{Introduction}

Wind turbines using self-excited three-phase induction generators are relatively widespread as devices used to produce electrical energy at remote sites when the required power is relatively limited. This choice is the result of a compromise which favors the structure's robustness relative to the regulation facilities [1]. These wind turbines, which are often used in conjunction with diesel groups $[2,3]$, are typically used at private (or a whole of private) and technical sites [4]. However, more and more, there exists a reluctance to use these devices because the use of these generators, for these specific applications, results in rather poor control. This can be explained by the lack of analytical expressions which make it possible to characterize in a simple way the influence of various parameters on the system behavior in the steady state. The great majority of approaches used are therefore numerical ones, [5-7], leading to properties which cannot systematically come into general use. These numerical approaches are all the more justified since the use of assumptions that are too simplified distort the problem leading, sometimes, to mistakes in the interpretation of the operational mode of the wind turbines. To surmount these difficulties it is

\footnotetext{
a e-mail: remus.pusca@univ-artois.fr
}

possible to defer the problem onto the regulation facilities and their techniques, which are then relatively complex [8-10]. However, this solution is not easy to implement in some critical sites due to the climatic conditions and (or) the availability of skilled labour in a geographical environment sufficiently nearby [11].

The main reasons at the origin of these difficulties result from the inverse nature of the problem to be solved (frequency, slip and voltage unknown) and due to the fact that it is based on the traditional equivalent single-phase circuit of the Induction Machine (IM) [6,12], which, from the beginning, has been conceived to function as a motor.

The study intends to exploit a single-phase equivalent circuit that more appropriately describes the steady state operation of a three-phase IM acting as a self-excited generator requiring a connection at the stator outputs, in addition to the load, a capacitor bank. After presenting this equivalent circuit as well as the relationships which govern its operation, the analysis will be carried out by considering first a resistive load. The objectives concern the definition of reliable angular frequency and slip analytical expressions of a $\underline{\text { Self-Ex}} \mathbf{E} x i t e d \underline{I n d u c t i o n} \underline{\mathbf{G}}$ enerator (SEIG) which then also permit an easy analysis taking into account all parameters which define the IM. Nevertheless, this characterization induces a constraint because these expressions cannot cover all the possible operating 


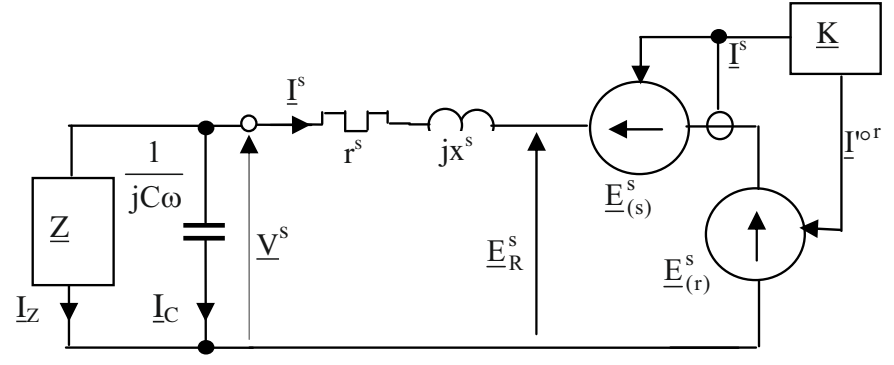

Fig. 1. Single-phase equivalent circuit of the SEIG.

ranges of this SEIG. So, the concept of a controlled operating zone will be introduced. It will be shown that, in this zone, these analytical expressions make it possible to define a very simple strategy for voltage regulation. This strategy, not only protects the SEIG from dangerous high current and torque transients during load state changes, but also avoids the loss of SEIG control due to its disengagement. This voltage control leads then to operation at a quasi constant frequency so that, from the load, the SEIG runs practically like a synchronous generator without a frequency control loop. Finally, elementary considerations - extrapolating the results from the study considering a resistive load - allow one to propose a very simple control strategy leading to a quasi constant voltage-frequency operation, valid when the nature of the load changes.

\section{Presentation of the single phase equivalent circuit}

The single-phase equivalent circuit of a $p$ pole pair IM, based on the concept of an induced voltage source $[13,14]$ when the iron losses are neglected, is given in Figure 1. In this figure also appears the load $\underline{\mathrm{Z}}$ connected to the stator outputs and, in addition, a capacitor of capacitance $C$ used to provide the reactive power necessary, on one hand, for the magnetization of the IM and, on the other hand, for the load consumption.

Let us assume that the stator windings are crossed by a sinusoidal, balanced, three-phase current system of angular frequency $\omega$ (frequency $f$ ). The synchronous speed is defined by: $\Omega=\omega / p$. The angular rotor speed $\Omega^{\prime}$ is equal to: $\Omega^{\prime}=(1-s) \Omega$, where $s$ denotes the slip. Operation as a generator requires that: $\Omega^{\prime}>\Omega$, therefore $s<0$. As saturation is neglected, the equations which describe the SEIG operation are expressed as:

$$
\left.\begin{array}{c}
\underline{V}^{s}=r^{s} \underline{I}^{s}+j x^{s} \underline{I}^{s}+\underline{E}_{R}^{s} \\
\underline{E}_{R}^{s}=\underline{E}_{(s)}^{s}+\underline{E}_{(r)}^{s} \\
\underline{E}_{(s)}^{s}=j X^{s} \underline{I}^{s} \\
\underline{E}_{(r)}^{s}=j X^{s} \underline{I}^{\circ r} \\
\underline{E}_{R}^{s}=j X^{s} \underline{I}_{R}^{s}
\end{array}\right\}
$$

with:

$$
\left.\begin{array}{c}
\underline{I}_{R}^{s}=\underline{I}^{s}+\underline{I}^{\prime o r} \\
\underline{I}^{\prime \circ}=\underline{K I}^{s}=-j L^{s} s \omega /\left[r^{\prime r}+j\left(L^{s}+l^{\prime r}\right) s \omega\right]
\end{array}\right\} .
$$

$-X^{s}=L^{s} \omega, x^{s}=l^{s} \omega$, with $L^{s}$ the main cyclic self inductance and $l^{s}$ the leakage inductance of the stator windings,

$-x^{\prime r}=l^{\prime r} \omega$ with $l^{\prime r}$ the leakage inductance of the rotor copper arrangement (wound or cage rotor) referred to the stator side,

$-r^{s}$ is the stator winding resistance,

$-r^{\prime r}$ is the rotor copper arrangement resistance referred to the stator side.

To characterize these leakage inductances, they will be defined relative to $L^{s}: l^{s}=\lambda^{s} L^{s}, l^{\prime r}=\lambda^{r} L^{s}$ where $\lambda^{s}$ and $\lambda^{r}$ are constants whose values are small relative to unity. Let us point out that the quantities $l^{s}, l^{\prime r}$ as well as $\mathrm{r}^{s}$ will be referred to as parasitical terms in the following work.

Equations (1), (2) and those of system (3) are similar in their forms to those which characterize the smooth air gap synchronous machine. However, for the synchronous machine, the rotor current associated with $\underline{I}^{\circ}$ is an external quantity, controlled by the operator, and is independent of the stator currents which, in turn, are dependent on the rotor current. Consequently, the stator voltage equation alone is sufficient to define the single-phase synchronous machine equivalent circuit. For the IM, the two currents $\underline{I}^{s}$ and $\underline{I}^{\prime \circ r}$ depend on each other according to the equations of system (5).

The circuit given in Figure 1 leads to the time phasor diagram presented in Figure 2. Considering the projections along the $x$ and $y$ axes, which correspond to the active and reactive powers, one obtains:

$$
\left.\begin{array}{c}
E_{(r)}^{s} \cos \left(\varphi^{\prime s}-\varphi^{r}\right)+E_{(s)}^{s} \sin \varphi^{\prime s} \\
-r^{s} I^{s} \cos \varphi^{\prime s}+x^{s} I^{s} \sin \varphi^{\prime s}=V^{s} \\
E_{(r)}^{s} \sin \left(\varphi^{\prime s}-\varphi^{r}\right)-E_{(s)}^{s} \cos \varphi^{\prime s} \\
-r^{s} I^{s} \sin \varphi^{\prime s}-x^{s} I^{s} \cos \varphi^{\prime s}=0
\end{array}\right\} .
$$

- The quantity $-\varphi^{\prime s}$, which represents the phase lag of $-\underline{I}^{s}$ relative to $\underline{V}^{s}$, is tied to the load and the capacitor connected to the stator outputs.

- $\varphi^{r}$ corresponds to the $\underline{K}$ denominator angle: $\varphi^{r}=$ $\arctan \left[\left(L^{s}+l^{\prime r}\right) s \omega / r^{\prime r}\right]$. Considering the second equation of system (3), which can equally be written as: $\underline{E}_{(r)}^{s}=j X^{s} \underline{K I}^{s}$, and taking that $s$ is negative into account, it is noted that $-\varphi^{r}$ also gives the phase angle of $\underline{E}_{(r)}^{s}$ relative to $-\underline{I}^{s} \cdot \varphi^{r}$, which evolves between 0 and $-\pi / 2$, is characterized by:

$$
\left.\begin{array}{l}
\sin \varphi^{r}=s\left(X^{s}+x^{\prime r}\right) / H \\
\cos \varphi^{r}=r^{\prime r} / H \\
H=\sqrt{r^{\prime r 2}+s^{2}\left(X^{s}+x^{\prime r}\right)^{2}}
\end{array}\right\} .
$$

$-\gamma^{r}$, corresponds to the phase angle of $\underline{I}^{\prime \circ r}$ relative to $\underline{E}_{R}^{s} \cdot \gamma^{r}$ expression results from $\underline{E}_{R}^{s}$ which can also be expressed as: $\underline{E}_{R}^{s}=j X^{s}[(1+\underline{K}) / \underline{K}] \underline{I}^{\prime \circ}$. Calculations show that $\gamma^{r}$ must take values between 0 and $\pi / 2$. 


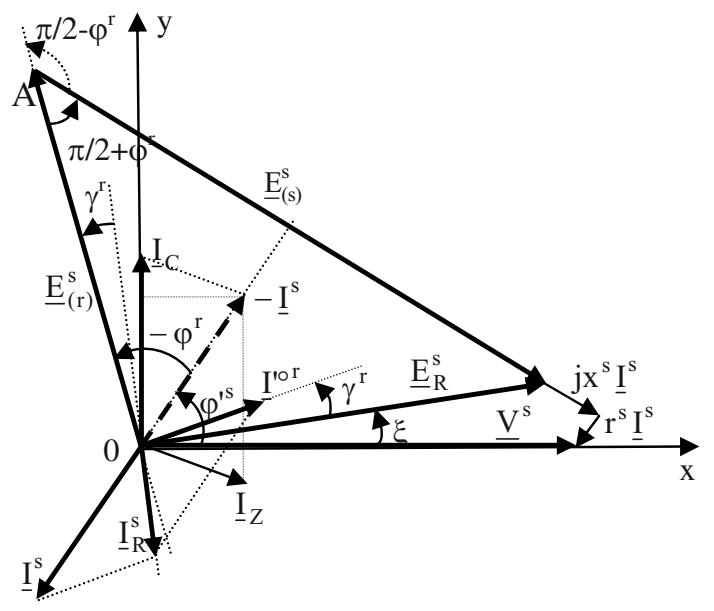

Fig. 2. Time phasor associated with the SEIG single-phase equivalent circuit.

- $\xi$ denotes the phase angle of $\underline{E}_{R}^{s}$ relative to $\underline{V}^{s}$.

One may notice that equations (1) to (7) are valid regardless of the load. In the following, the load will be first considered as resistive.

\section{Relationships for a resistive load}

If this application considering only a resistive load seems restrictive, one should note that it is for this type of application that a majority of such SEIGs are used. Voltage regulation can be carried out simply by connecting or disconnecting resistive elements. The study of this elementary case is nevertheless complex due to the inverse nature of the problem which has as its objective to determine analytical expressions for $\omega, s$ (so $\Omega^{\prime}$ ) and $V^{s}$ when an assumed wind power $P_{w}$ is transmitted on the SEIG shaft. If $R$ denotes the equivalent load impedance, so: $\underline{Z}=R$.

\subsection{Voltage relationships}

$-\varphi^{\prime s}$ is characterized by:

$$
\left.\begin{array}{l}
\sin \varphi^{\prime s}=R C \omega / G \\
\cos \varphi^{\prime s}=1 / G \\
G=\sqrt{1+R^{2} C^{2} \omega^{2}}
\end{array}\right\}
$$

This phase angle evolves between 0 and $\pi / 2$. The rms voltage results from:

$$
V^{s}=R I^{s} / G .
$$

Let us substitute these quantities in the equations of system (6). Taking into account the expression for $\varphi^{r}$ and introducing the constants:

$$
\left.\begin{array}{rl}
A^{\prime \prime}= & L^{s 2}\left(1+\lambda^{r}\right)\left\{L^{s}\left[1-\left(1+\lambda^{s}\right)\left(1+\lambda^{r}\right)\right]\right. \\
& \left.-r^{s} R C\left(1+\lambda^{r}\right)\right\} \\
B= & L^{s 2} R C r^{\prime r} \\
D^{\prime \prime}= & r^{\prime r 2}\left\{L^{s}\left(1+\lambda^{s}\right)+r^{s} R C\right\} \\
E^{\prime \prime}= & L^{s 2}\left(1+\lambda^{r}\right)^{2}\left[R+r^{s}\right]
\end{array}\right\}
$$

leads one to characterize the SEIG operating mode by the following equations:

$$
\left.\begin{array}{c}
R C A^{\prime} s^{2} \omega^{4}+E^{\prime \prime} s^{2} \omega^{2}+L^{s 2} r^{\prime r} s \omega^{2} \\
-D^{\prime} R C \omega^{2}+\left(r^{s}+R\right) r^{\prime r 2}=0 \\
A^{\prime \prime} s^{2} \omega^{2}-B s \omega^{2}-D^{\prime \prime}=0
\end{array}\right\} .
$$

The constants denoted by the superscript "," result from those denoted with the superscript " " by considering the case $r^{s}=0$. Let us point out that the constants $A^{\prime \prime}$ and $A^{\prime}$ are negative quantities whereas all the other terms are positive.

\subsection{Electromagnetic torque}

The IM electromagnetic torque results from the cross product: $\Gamma_{e}=\kappa^{\prime} \vec{b}_{p}^{s} \times{\overrightarrow{b^{\circ}}}_{p}^{r}$ where $\kappa^{\prime}$ is a constant tied to the IM parameters. $\vec{b}_{p}^{s}$ and $\vec{b}_{p}^{r}$ are the air gap flux density space phasors, generated by the stator and rotor windings respectively, defined in the stator reference frame. As $\underline{E}_{(s)}^{s}$ and $\underline{E}_{(r)}^{s}$ come from $\vec{b}_{p}^{s}$ and $\overrightarrow{b^{\circ}} \underset{p}{r}$, respectively, using the same procedure of integration and derivation, it results that $\Gamma_{e}$ can also be expressed as:

$$
\Gamma_{e}=\kappa \vec{E}_{(s)}^{s} \times \vec{E}_{(r)}^{s}=\kappa E_{(s)}^{s} E_{(r)}^{s} \sin \left(\frac{\pi}{2}-\varphi^{r}\right)
$$

where: $\kappa=3 p / X^{s} \omega$. The use of equation (12) makes it possible to express $V^{s}$ in terms of $\Gamma_{e}$ :

$$
V^{s}=\frac{R}{L^{s}} \sqrt{\frac{\Gamma_{e}}{3 p \omega} \frac{r^{\prime r 2}+s^{2}\left(X^{s}+x^{\prime r}\right)^{2}}{\left(1+R^{2} C^{2} \omega^{2}\right) s r^{\prime r}}}
$$

where $\Gamma_{e}$ is related to $P_{w}$ using the relationship: $P_{w}=$ $\left(\Gamma_{e}+\Gamma_{f}\right) \Omega^{\prime}$, where $\Gamma_{f}$ represents the IM friction and windage torque. Let us note that equation (13) is the same one as that which can be deduced from the traditional IM single-phase equivalent circuit.

\section{Development of an analytical solution}

The equations of system (11) show that $\omega$ and $s$ depend only on $R, C$ and the parameters which characterize the IM. So, for given $R, C$ and IM, the SEIG will be locked to a constant operating angular frequency regardless of $P_{w}$. This property is also true for the slip. Indirectly this means that $\Omega^{\prime}$ will be fixed, and only $V^{s}$ will be influenced by the torque applied on the SEIG shaft. The aim of this analysis is to determine analytic expressions for $\omega$ and $s$ in order to be able to determine easily the SEIG behavior, and in particular:

- to appreciate the impact of parasitic terms on the $\underline{\text { Operating }} \underline{\text { Point }}(\mathbf{O P})$ definition,

- to define the ranges of $R$ and $C$ which lead to controlled operation,

- to suggest solutions in order to stabilize operation during a transient state using voltage control by changing the value of $R$. 


\subsection{Procedure of study}

The analytical solutions of system (11) which characterize the accurate expressions $\omega_{e x}$ and $s_{e x}$ are in very complex forms. They therefore cannot be used to address the previous items, even in approximate form. It would be conceivable to numerically exploit these equations. However, taking into account the relatively high number of parameters, it is foreseeable that it will be difficult to deduce some general laws. Consequently it is necessary to use another approach which consists of determining approximate analytical solutions in the following form:

$$
\left.\begin{array}{l}
\omega \cong \omega_{\text {ref }}\left(1+\varepsilon_{(\omega)}\right) \\
s \cong s_{\text {ref }}\left(1+\varepsilon_{(s)}\right)
\end{array}\right\}
$$

If $\varepsilon_{(\omega)}$ and $\varepsilon_{(s)}$ are sufficiently small relative to unity, it will be possible that the analytic expressions for $\omega_{\text {ref }}$ and $s_{\text {ref }}$ are representative of the SEIG behavior leading, under these conditions, to reduce the problem to the determination of these quantities. The numerical values which will be deduced from $\omega_{\text {ref }}$ and $s_{\text {ref }}$ could be improved by calculating the correction terms $\varepsilon_{(\omega)}$ and $\varepsilon_{(s)}$ which correspond to estimated precisions. In order to test the validity of this procedure, the relative errors $e_{(\omega)}$ and $e_{(s)}$ will also be calculated for a given $R$ and $C$ :

$$
\left.\begin{array}{l}
e_{(\omega)}=\left(\omega_{e x}-\omega_{r e f}\right) / \omega_{e x} \\
e_{(s)}=\left(s_{e x}-s_{r e f}\right) / s_{e x}
\end{array}\right\}
$$

Using the numerical values deduced from the analytic expressions for $\omega_{\text {ref }}$ and $s_{\text {ref }}$ to illustrate this methodology, applications will be presented on an IM characterized by: $220 / 380 \mathrm{~V}, 6.2 / 3.6 \mathrm{~A}, \cos \varphi^{s}=0.85,50 \mathrm{~Hz}, 1410 \mathrm{rpm}$, $1.7 \mathrm{~kW}$. Standard tests lead to: $L^{s}=400 \mathrm{mH}, r^{s}=5.35 \Omega$, $r^{\prime r}=3.6 \Omega, l^{s}+l^{\prime r}=33 \mathrm{mH}$. It will be assumed that: $l^{s}=15 \mathrm{mH}, l^{\prime r}=18 \mathrm{mH}$ so that: $\lambda^{s}=3.75 \%, \lambda^{r}=4.5 \%$. Rated values (subscript "rated") make it possible to estimate: $\Gamma_{e(\text { rated })}=11.5 \mathrm{Nm}, s_{(\text {rated })}=6 \%$. Moreover, to obtain operation corresponding to the rated power under $V_{(\text {rated })}^{s}=220 \mathrm{~V}$ for $\omega_{(\text {rated })}=314.1 \mathrm{rad} / \mathrm{s}\left(\Omega_{(\text {rated })}=\right.$ $157 \mathrm{rad} / \mathrm{s}), R$ must be in the range of $R_{(\text {rated })}=80 \Omega$.

\subsection{First approach neglecting the parasitic terms}

When the parasitic terms are neglected, $\omega$ and $s$ can be equated to $\omega_{0}$ and $s_{0}$, as defined by:

$$
\left.\begin{array}{l}
\omega=\omega_{0}=1 / \sqrt{L^{s} C} \\
s=s_{0}=-r^{\prime r} / R
\end{array}\right\}
$$

leading one to express $V^{s}$ as follows:

$$
V^{s}=\sqrt{-R \Gamma_{e} \omega / 3 p} .
$$

That implies, when the parasitic terms are taken into account, that $\omega_{\text {ref }}$ and $s_{\text {ref }}$ which intervene in the equations of system (14), can be chosen to be equal to $\omega_{0}$ and $s_{0}$. In order to obtain an angular frequency close to $\omega_{(\text {rated })}$
Table 1. Influence of the parasitic terms for $R=R_{0}, C=C_{0}$.

\begin{tabular}{ccccc}
\hline$r^{s}$ & $\lambda^{s}$ & $\lambda^{r}$ & $\omega_{e x}(\mathrm{rad} / \mathrm{s})$ & $s_{\text {ex }}(\%)$ \\
\hline 0 & 0 & 0 & $314(30.2 \%)$ & $-6.00(8.8 \%)$ \\
5.35 & 0 & 0 & $342(24 \%)$ & $-5.62(14.5 \%)$ \\
0 & $3.75 \%$ & 0 & $339(24.7 \%)$ & $-6.51(0.9 \%)$ \\
5.35 & $3.75 \%$ & 0 & $372(17.3 \%)$ & $-6.12(6.8 \%)$ \\
0 & 0 & $4.5 \%$ & $351(22 \%)$ & $-6.07(7.6 \%)$ \\
5.35 & 0 & $4.5 \%$ & $386(14.2 \%)$ & $-5.72(12.9 \%)$ \\
0 & $3.75 \%$ & $4.5 \%$ & $400(11.1 \%)$ & $-6.85(-4.3 \%)$ \\
5.35 & $3.75 \%$ & $4.5 \%$ & $450(0 \%)$ & $-6.57(0 \%)$ \\
\hline
\end{tabular}

with a slip close to $-s_{(\text {rated })}$, it is first assumed that $\varepsilon_{(\omega)}$ and $\varepsilon_{(s)}$ are small relative to the unit. It is thus possible to replace $\omega$ with $\omega_{0}$ and $s$ with $s_{0}$ to calculate $R$ and $C$. In these conditions, one obtains: $C=C_{0}=25.33 \mu \mathrm{F}$, $R=R_{0}=60 \Omega$. Thus, the numerical values of the constants for the system $(10)$ are: $A^{\prime}=-5.63 \times 10^{-3}$, $A^{\prime \prime}=-7.05 \times 10^{-3}, B=0.875 \times 10^{-3}, D^{\prime}=5.38$, $D^{\prime \prime}=5.48, E^{\prime}=10.48, E^{\prime \prime}=11.42$. Using these quantities, one obtains: $\omega_{e x}=450 \mathrm{rad} / \mathrm{s}, s_{e x}=-6.57 \%$, which leads to: $e_{(\omega)}=43.3 \%, e_{(s)}=9.5 \%$. These values, in particular that of $e_{(\omega)}$, are much too large to be able to assume that $\omega_{0}$ and $s_{0}$ are representative of an approximate analytical solution. Consequently, another procedure must be defined. Nevertheless, at this stage of the analysis, it seems interesting to appreciate the impact of the parasitic terms on the $\omega_{e x}$ and $s_{e x}$ values. The results obtained are grouped together in Table 1. In this table are also presented the relative discrepancies (numbers between brackets) of $\omega_{e x}$ and $s_{e x}$ defined relative to these same quantities calculated when all parasitical terms are considered.

An analysis of this table reveals the considerable part played by these parasitic terms on the $\mathbf{O P}$ definition. It must be specified that by first using the equalities: $\omega_{\text {ref }}=\omega_{0}, s_{\text {ref }}=s_{0}$, allows one, through successive iterations [15], to find values of $\omega$ and $s$ which are practically identical to $\omega_{e x}$ and $s_{e x}$. Nevertheless, as specified previously, this numerical procedure does not correspond to our aim which consists of determining approximate but reliable analytical expressions of the solution.

\subsection{Second approach using the minimal angular frequency}

Let us consider the second equation of system (11). It can be written as:

$$
s=B\left[1 \pm \sqrt{1+4 A^{\prime \prime} D^{\prime \prime} / B^{2} \omega^{2}}\right] / 2 A^{\prime \prime}
$$

requiring, as the quantity under the radical must be null or positive, to satisfy the inequality:

$$
\omega^{2} \geqslant \omega_{\min }^{2}=-4 A^{\prime \prime} D^{\prime \prime} / B^{2} .
$$

If $\omega$ is sufficiently close to $\omega_{\text {min }}$, equation (18) leads to:

$$
s \cong s_{\min }=B / 2 A^{\prime \prime}
$$


The numerical application using the numerical values specified in Section 4.1 gives: $\omega_{\min }=449.3 \mathrm{rad} / \mathrm{s}$, $s_{\text {min }} \cong-6.21 \%$. Let us note that $\omega_{\min }$ is practically identical to $\omega_{e x}$ whereas $s_{\text {min }}$ presents a value more distant from $s_{e x}$ but with less discrepancy than that which characterizes this quantity as determined through the first approach. This particularity concerning the angular frequency, encourages one to adopt as reference variables the quantities $\omega_{m i n}$ et $s_{\text {min }}$, leading to: $e_{(\omega)}=0.16 \%$, $e_{(s)}=5.8 \%$. However, this choice does not rely on any physical consideration, so the obtained numerical values may have resulted quite simply from a coincidence. It is thus advisable to determine within which limits this result can come into general use. With this aim in view, system (11) will be exploited through graphical means.

\section{Graphical analysis}

\subsection{Principle}

- Let us consider the first equation of system (11). Noting:

$$
\left.\begin{array}{l}
M=R C A^{\prime} s^{2} \\
N=E^{\prime \prime} s^{2}+L^{s 2} r^{\prime r} s-D^{\prime} R C \\
P=\left(r^{s}+R\right) r^{\prime 2}
\end{array}\right\}
$$

leads to a quadratic equation in $\omega^{2}$ whose roots are expressed by:

$$
\omega^{2}=-N\left[1 \mp \sqrt{1-4 M P / N^{2}}\right] / 2 M .
$$

Let us note that $\omega_{11}$ is the value of $\omega$ which results from the previous equation defined with a negative sign in front of the radical, and $\omega_{12}$ is that defined with a positive sign. As $M$ is negative, the quantity under the radical of (22) is greater than unity. The $\omega_{11}$ value will be defined only if $N$ is negative whereas for $\omega_{12}$ to exist, $N$ must be positive. Let us denote $s_{\text {crit }}$ as the negative value of $s$ which cancels out $N$ :

$$
s_{c r i t}=-\left[L^{s 2} r^{\prime r}+\sqrt{L^{s 4} r^{\prime r 2}+4 D^{\prime} R C E^{\prime \prime}}\right] / 2 E^{\prime \prime} .
$$

It can be deduced that $\omega_{11}$ only exists for the range $s_{\text {crit }}<$ $s<0$, and when $s$ tends towards 0 from the negative side: $\omega_{11}^{2}=\omega_{0}^{2}\left(R+r^{s}\right) / R\left(1+\lambda^{s}\right)$. Neglecting $r^{s}$ relative to $R$ and $\lambda^{s}$ relative to 1 , it appears that in this limit, the value of $\omega_{11}$ is equal to $\omega_{0}$. For a value of $s$ smaller than $s_{\text {crit }}$, it is $\omega_{12}$ that it is advisable to consider. When $s$ tends towards $s_{\text {crit }}$ (with $s<s_{\text {crit }}$ for $\omega_{12}$ and $s>s_{\text {crit }}$ for $\left.\omega_{11}\right)$, by noting: $M_{c r i t}=R C A^{\prime} s_{c r i t}^{2}, \omega_{11}$ and $\omega_{12}$ tend both towards a single value $\omega_{\text {crit }}$ defined by:

$$
\omega_{\text {crit }}=\left(-P / M_{\text {crit }}\right)^{1 / 4} \text {. }
$$

- Taking into account the second equation of system (11) leads to:

$$
\omega^{2}=D^{\prime \prime} /\left[A^{\prime \prime} s^{2}-B s\right] .
$$

Let us denote as $\omega_{2}$ the positive value of $\omega$ which results from (25). According to the $s_{\min }$ definition given by (20),

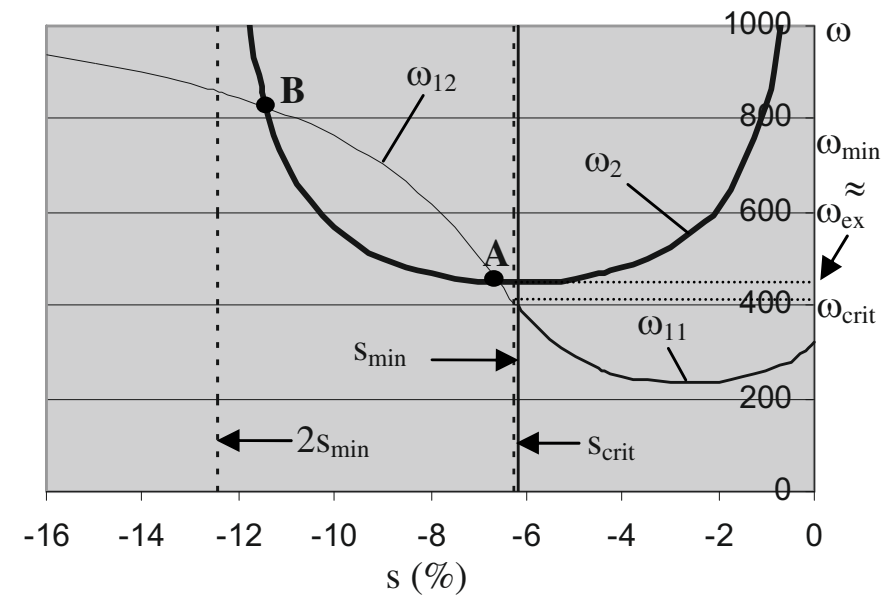

Fig. 3. Operating point positions for $R=R_{0}, C=C_{0}$.

this term only exists for: $2 s_{\min }<s<0$. $\omega_{2}$ tends towards infinity when $s$ tends towards 0 and $2 s_{\text {min }}$. Generally, the possible OPs are defined by the intersections of the $\omega_{11}(s)$ and $\omega_{12}(s)$ curves with that of $\omega_{2}(s)$. Consequently, it can be deduced that these $\mathbf{O P s}$, if they exist, are characterised by the slips that satisfy the condition:

$$
s \in] 2 s_{\min } ; 0[\text {. }
$$

- Figure 3 presents the variations of $\omega_{11}$ (fine, solid curve), $\omega_{12}$ (fine, dashed curve) and $\omega_{2}$ (bold curve) with $s$ for the previously described IM associated with $R_{0}$ and $C_{0}$. According to condition (26), the allowed range of $s$ is restricted to: $s=[-0.16 ; 0]$. It can be noted from this figure there exist two possible OPs (points $A$ and $B$ ) which result only from the intersections of the curves $\omega_{12}(s)$ and $\omega_{2}(s)$.

The $A$ OP, whose coordinates correspond to the quantities $\omega_{e x}$ and $s_{e x}$ as specified previously $(450 \mathrm{rad} / \mathrm{s} ;-6,57 \%)$, is located in the part of the $\omega_{2}(s)$ curve where the variations of $\omega$ with $s$ are relatively restricted. The fact that this $\mathbf{O P}$ is in this zone which can be described as stable, justifies the accuracy and the uncertainty which affect the values of $\omega$ and $s$ respectively as mentioned in Section 4.3. For given $\Gamma_{e}$, equation (17) - which defines a simplified expression of $V^{s}$ - shows that the voltage will be consequently very little affected by natural variations (saturation, heating effects...) in the parameters which intervene in the IM equivalent circuit definition.

The $B$ OP leads to relatively high values of $|s|$ and $\omega$ $(11.4 \%, 826 \mathrm{rad} / \mathrm{s})$, thus damaging energetic performance of the SEIG. This OP is also characterized by a much larger inaccuracy in $\omega$ due to the natural variations in the IM parameters which directly modify the value of $V^{s}$.

Considering the derivative of $\omega_{2}$ versus $s$, one can note that it is null when: $s=s_{\min }$. The two peaks leading to infinite $\omega_{2}$ are consequently symmetrically located relative to this minimum. The vertical portions of the dashed line correspond to $s_{\min }(-6,21 \%)$ and $2 s_{\min }$. The intersection of the vertical line at $s_{\min }$ with the curve $\omega_{2}(s)$ defines $\omega_{\min }(449.3 \mathrm{rad} / \mathrm{s})$, as given by (19), which is, as specified 
previously, very close to $\omega_{e x} . s_{\text {crit }}$, which results from (23), is equal to $-6.20 \%$ defining, from $(24), \omega_{\text {crit }}=400.6 \mathrm{rad} / \mathrm{s}$. The continuous vertical line corresponding to $s_{\text {crit }}$, practically identical with the as defined value of $s_{\text {min }}$, delimits two areas characterized by OPs which belong to the characteristics $\omega_{11}(s)$ or $\omega_{12}(s)$. The angular frequency of transition is equal to $\omega_{\text {crit }}$. The difference $\delta=\omega_{\text {min }}-\omega_{\text {crit }}$ is equal to $48.7 \mathrm{rad} / \mathrm{s}$.

\subsection{Variations of $\mathrm{R}$ and of $\mathrm{C}$}

- Figure 4 shows similar variations as above, keeping the value of $C$ constant at $C_{0}$, but giving $R$ values of $55 \Omega$ (Fig. 4a) and $75 \Omega$ (Fig. 4b). The numerical values of $\omega_{e x}$, $s_{\text {ex }}, \omega_{\text {min }}, s_{\text {min }}, \omega_{\text {crit }}$ and $s_{\text {crit }}$ obtained in these conditions are grouped together in Table 2, where the quantities obtained for $R_{0}$ are also presented.

For $R=55 \Omega$, the $A \mathbf{O P}$, which results from the intersection of the curves $\omega_{12}(s)$ and $\omega_{2}(s)$, presents a slip which moves away from $s_{\text {crit }}$ with a much larger difference relative to $s_{\min }$. It results that the precision of $\omega$, defined by $\omega_{\text {ref }}=\omega_{\text {min }}$, is reduced as long as the $A$ OP exists in the curved parts of the stable zone. This particularity increases the $\delta$ value which is equal to $95.1 \mathrm{rad} / \mathrm{s}$. Further decreasing more the value of $R$ leads to a single operating mode as the $A$ and $B$ OPs are co-located (curves $\omega_{12}(s)$ and $\omega_{2}(s)$ tangent). This occurs for a $R^{*}$ value of $R$ which is equal to $52.5 \Omega$ (the numerical values for this $\mathbf{O P}$ are given in Tab. 2). For values of $R$ lower than $R^{*}$ there is no possible $\mathbf{O P}$.

When $R$ increases and takes on a value of $75 \Omega$, and considering again $\mathbf{O P} A$, it appears that this last operating point results from the intersection of the curves $\omega_{11}(s)$ and $\omega_{2}(s)$. The accuracy in the slip compared to $s_{c r i t}$ is clearly improved. On the other hand, compared to the previous case $(R=55 \Omega)$, the difference compared to $s_{\text {min }}$ not only changes in sign but also increases in absolute value. $\delta$ takes a negative value $-56,7 \mathrm{rad} / \mathrm{s}$. The three variables $\omega_{e x}, \omega_{\text {min }}$ and $\omega_{\text {crit }}$ (see Tab. 2) present similar values. In this case, there are no limits to increasing the value of $R$ except those inherent to the energetic performance of the SEIG, and to the voltage stability.

- For a given value of $R$ equal to $R_{0}$, the initial value of $C_{0}$ has also been increased and decreased by about $20 \%$. An analysis of the results shows that the conclusions are similar to those formulated previously concerning the changes in $R$. In this case, the minimal value $C^{*}$ of $C$ (where the $A$ and $B$ OPs merged) is $19.1 \mu \mathrm{F}$. For lower values of $C$ there are no possible OPs. The various values of the angular frequencies and the slips are also grouped together in Table 2.

This graphical analysis stresses the very marked nonlinear character of the SEIG behaviour which allows one to foresee certain difficulties of control if no precautions are taken.

\section{Analysis of the graphical study}

\subsection{Approximate expressions of the variables}

The previous analysis shows, concerning $\mathbf{O P} A$, that $\omega_{\text {ref }}$ and $s_{\text {ref }}$ can be, with sufficient coherence, given respectively by $\omega_{\min }$ (Eq. (19)) and by $s_{\text {crit }}$ (Eq. (23)), rather than by $s_{\min }$ as was initially done in Section 4.3 . These expressions will be valid only on the condition that $R$ and $C$ do not take values which place $\mathbf{O P} A$ too far into in the curved section of the stable characteristic $\omega_{2}(s)$, for previously evoked reasons. Taking the constant expressions given by (10) into account, leads to:

$$
\left.\begin{array}{l}
\omega_{\text {ref }}^{2}=\omega_{\min }^{2} \\
=4 \frac{\omega_{0}^{2}}{R^{2}}\left(1+\lambda^{r}\right)\left\{\frac{L^{s}}{C}\left(1+\lambda^{s}\right)\left[\left(1+\lambda^{r}\right)\left(1+\lambda^{s}\right)-1\right]\right. \\
\left.+r^{s} R\left[2\left(1+\lambda^{r}\right)\left(1+\lambda^{s}\right)-1\right]+\frac{r^{s 2} R^{2} C}{L^{s}}\left(1+\lambda^{r}\right)\right\} \\
s_{\text {ref }}=s_{\text {crit }}=-\frac{r^{\prime r}}{2\left(1+\lambda^{r}\right)^{2}\left(R+r^{s}\right)} \\
\quad \times\left\{1+\sqrt{1+4 \omega_{0}^{2}\left(1+\lambda^{s}\right)\left(1+\lambda^{r}\right)^{2} R C^{2}\left(R+r^{s}\right)}\right\}
\end{array}\right\} .
$$

These equations show that $\omega_{\text {ref }}$ is independent of $r^{\prime r}$ whereas $s_{r e f}$ is proportional to it. This property also appears when the traditional equivalent circuit for the SIEG is used. Concerning the parasitical terms, it appears that the leakage inductances intervene in a predominant manner in the analytical definition of these variables. In particular, the major contribution of the rotor leakage inductance should be noted.

\subsection{Characterization of the controlled operation regime}

The aim is to define within which limits $R$ and $C$ may vary so as to be able to characterize $\omega$ and $s$ with sufficient accuracy using the $\omega_{\text {ref }}$ and $s_{\text {ref }}$ expressions defined by (27). To achieve this, three criteria are implemented.

- Criterion 1: Energetic performance, voltage stability. To simultaneously satisfy the constraints tied to these two aspects, the OP must be in the stable zone of $\omega_{2}(s)$ but not too far along in the curved section of the curve. Noting that the stable zone has a certain symmetry about $s_{\text {min }}$ and that the peaks which appear on this curve are far from $s_{\text {min }}$ on this symmetrical axis, it is sufficient that $R$ and $C$ have values such that:

$$
(1+k) s_{\min }<s_{\text {crit }}<(1-k) s_{\text {min }}
$$

where $k$ is a positive number less than unity. The smaller the value of $k$, the more equations (27) will be reliable, and therefore reducing the appropriate range for $R$ and $C$. For $C=C_{0}$ and $R=R^{*}$, the numerical values given previously show that: $s_{e x}=1.29 s_{\text {crit }}$. It can be deduced that $k=0.20$ is, a priori, a rather good compromise to both satisfy the initial requirements and to avoid the loss 


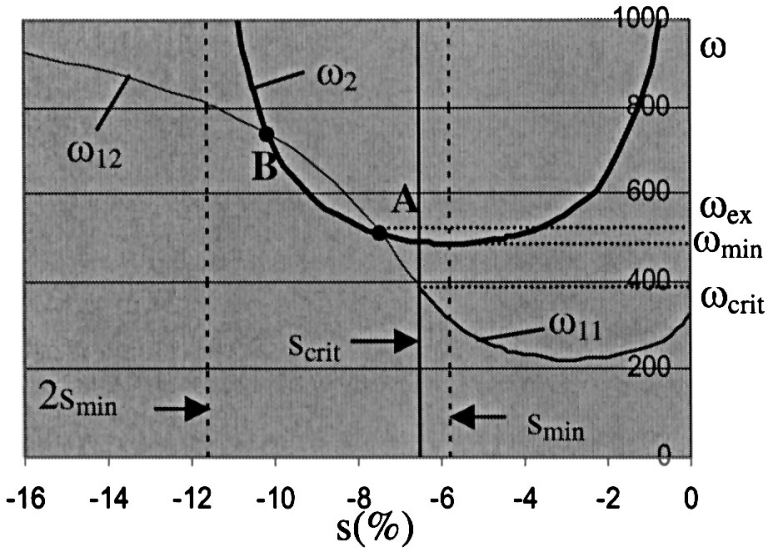

(a) $\mathrm{R}=55 \Omega, C=C_{0}$

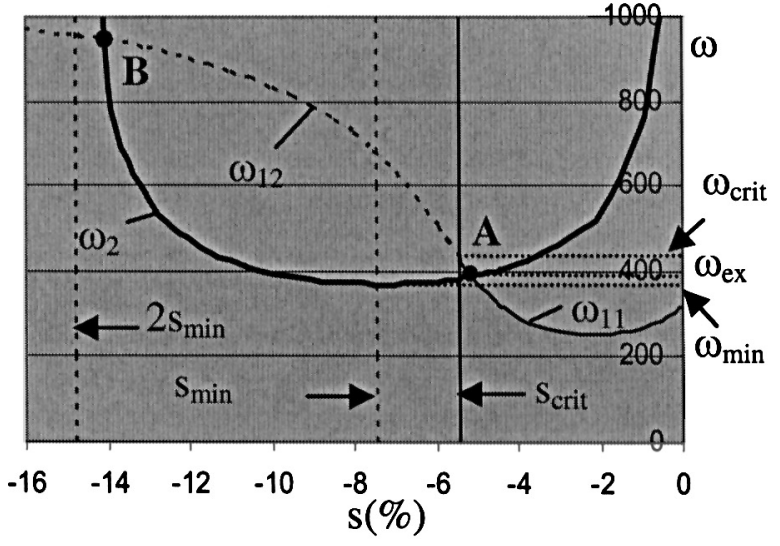

(b) $\mathrm{R}=75 \Omega, C=C_{0}$

Fig. 4. Operating point positions for variable $R$ and $C=C_{0}$.

Table 2. Angular frequencies and slips characterizing the operating points.

\begin{tabular}{cccccccc}
\hline$C(\mu \mathrm{F})$ & $R(\Omega)$ & $\omega_{\text {ex }}(\mathrm{rd} / \mathrm{s})$ & $s_{\text {ex }}(\%)$ & $\omega_{\min }(\mathrm{rd} / \mathrm{s})$ & $s_{\min }(\%)$ & $\omega_{\text {crit }}(\mathrm{rd} / \mathrm{s})$ & $s_{\text {crit }}(\%)$ \\
\hline & $R_{0}=60$ & 450 & -6.57 & 449.3 & -6.21 & 400.6 & -6.20 \\
$C_{0}=25.33$ & 55 & 507 & -7.45 & 485.6 & -5.79 & 390.5 & -6.55 \\
& $R^{*}=52.5$ & 612 & -8.70 & 506.4 & -5.57 & 385.1 & -6.75 \\
& 75 & 387 & -5.15 & 369.2 & -7.39 & 425.9 & -5.45 \\
20.33 & & 584 & -6.99 & 547.5 & -5.19 & 430.1 & -6 \\
30.33 & $R_{0}=60$ & 385 & -6.46 & 383.3 & -7.15 & 377.3 & -6.39 \\
$C^{*}=19.1$ & & 701 & -7.68 & 580.2 & -4.92 & 438.9 & -5.95 \\
\hline
\end{tabular}

of the $\mathbf{O P}$ for low values of $R$ and $C$, leading to the disengagement of the SEIG.

Let us point out that energetic performance are determined by two factors:

Slip $s$. The rotor copper losses increase with $|s|$, and it thus results that it must be avoided that $|s|$ takes excessive values. On the other hand, for low values of $|s|$, a given value of $C$ and a high value of $R$, simultaneously satisfying the two conditions of small $|s|$ and $R \gg R_{(\text {rated })}$, would lead to a SEIG OP close to a no-load OP, a condition synonymous with low efficiency.

Angular frequency $\omega$. Excessive values of $\omega$ would result in high iron losses in the stator core. Moreover, $\omega$ notably higher than $\omega_{\text {(rated })}$ and a low value of $|s|$, would lead to a too high value of $\Omega^{\prime}$, raising mechanical resistance problems. These excessive values of $\Omega^{\prime}$ also significantly increase the corresponding friction and windage losses. Let us note that this aspect was intentionally ignored in this work, as the primarily aim was only to present an approach to solve the described problem.

- Criterion 2: This second criterion relates to the numerical values of the quantities $\varepsilon_{(\omega)}$ and $\varepsilon_{(s)}$ which characterize the variables $\omega$ and $s$. Referring to equations (14), if $\varepsilon_{(\omega)}$ and $\varepsilon_{(s)}$ are small compared to unity, it is possible to neglect the second and higher order terms so that: $\omega^{n} \cong \omega_{r e f}^{n}\left(1+n \varepsilon_{(\omega)}\right), s^{n} \cong s_{r e f}^{n}\left(1+n \varepsilon_{(s)}\right)$. By substituting these expressions into equations (11), a system of two first order equations with two unknowns is obtained, which leads to:

$$
\left.\begin{array}{l}
\varepsilon_{(\omega)}=\left[K_{1} J_{3}-K_{3} J_{1}\right] /\left[K_{3} J_{2}-K_{2} J_{3}\right] \\
\varepsilon_{(s)}=\left[K_{2} J_{1}-K_{1} J_{2}\right] /\left[K_{3} J_{2}-K_{2} J_{3}\right]
\end{array}\right\}
$$

where:

$$
\begin{aligned}
J_{1}= & -\left[R C A^{\prime} s_{r e f}^{2} \omega_{r e f}^{4}+E^{\prime \prime} s_{r e f}^{2} \omega_{r e f}^{2}\right. \\
& \left.+L^{s 2} r^{\prime r} s_{r e f} \omega_{r e f}^{2}-D^{\prime} R C \omega_{r e f}^{2}+\left(r^{s}+R\right) r^{\prime r 2}\right] \\
J_{2}= & -\left[4 R C A^{\prime} s_{r e f}^{2} \omega_{r e f}^{4}+2 E^{\prime \prime} s_{r e f}^{2} \omega_{r e f}^{2}\right. \\
& \left.+2 L^{s 2} r^{\prime r} s_{r e f} \omega_{r e f}^{2}-2 D^{\prime} R C \omega_{r e f}^{2}\right] \\
J_{3}= & -\left[2 R C A^{\prime} s_{r e f}^{2} \omega_{r e f}^{4}+2 E^{\prime \prime} s_{r e f}^{2} \omega_{r e f}^{2}\right. \\
& \left.+L^{s 2} r^{\prime r} s_{r e f} \omega_{r e f}^{2}\right] \\
K_{1}= & {\left[A^{\prime \prime} s_{r e f}^{2} \omega_{r e f}^{2}-B s_{r e f} \omega_{r e f}^{2}-D^{\prime \prime}\right] } \\
K_{2}= & {\left[2 A^{\prime \prime} s_{r e f}^{2} \omega_{r e f}^{2}-2 B s_{r e f} \omega_{r e f}^{2}\right] } \\
K_{3}= & {\left[2 A^{\prime \prime} s_{r e f}^{2} \omega_{r e f}^{2}-B s_{r e f} \omega_{r e f}^{2}\right] }
\end{aligned}
$$

It has been previously specified that in the stable zone, the imprecision in $s$ was greater than that of $\omega$. In order to retain the values of $R$ and $C$, it will be adopted that $\varepsilon_{(\omega)}$ and $\varepsilon_{(s)}$ must be, in absolute values, lower than 10 and $20 \%$ respectively.

- Criterion 3: The suggested approach ignores how variations of $R$ and $C$ may modify the characteristics which give the variations of the angular frequency with $s$. 
However, it has appeared that the stable zone widens as these elements present higher values (see Fig. 4). This fact leads to the risk that one may obtain OPs satisfying the two previous criteria but with excessively large slips. Considerations on the rotor copper losses, lead one to adopt a maximum value of $s$ higher than $-10 \%$ as a third criterion.

\subsection{Determination of the controlled operation regime}

The determination of the controlled operation regime results from the numerical analysis of the preceding equations. In order to do this, $C$ is chosen as a fixed parameter while $R$ is varied. The principle is to collect, for a given $C$, the following quantities:

- $R^{*}$, which corresponds to the minimal value of $R$ below which there exist no intersections of the $\omega_{12}(s)$ and $\omega_{2}(s)$ characteristics. This quantity, defined by successive approximations, is obtained from the analysis of the difference $\omega_{2}-\omega_{12}$ which must be positive for all $s$ except for a single value (denoted $s^{*}$ ) where it must be null. This leads to the quantities: $\omega^{*}, s^{*}, \omega_{m i n}^{*}, s_{m i n}^{*}$, $\omega_{c r i t}^{*}$ and $s_{c r i t}^{*}$. It can be pointed out that $s^{*}$ and $\omega^{*}$, which result from the graphical analysis, correspond to the $s_{e x}$ and $\omega_{e x}$ values.

- The $R$ values satisfying the previous criteria and, for each of these values, the $\mathbf{O P}$ coordinates assumed to be defined by $\omega_{\min }$ and $s_{\text {crit }}$ as well as the quantities $\varepsilon_{(\omega)}$ and $\varepsilon_{(s)} \cdot \omega_{e x}$ and $s_{e x}$ have been also calculated for each $\mathbf{O P}$ that allows one to determine the $e_{(\omega)}$ and $e_{(s)}$ values.

- The value $R_{T}$ of $R$ which corresponds to the transition of the $\mathbf{O P}$ from the $\omega_{11}(s)$ characteristic to $\omega_{12}(s)$ or vice versa.

- The minimal value of $R, R_{L}$, which satisfies the given criteria. This analysis shows that $R_{L}$ results only from the non-satisfaction of the condition concerning $\varepsilon_{(s)}$.

- The maximum value of $R, R_{H 1}$, which satisfies the given criteria. The numerical study reveals that this quantity is primarily determined by the nonsatisfaction of the condition: $s_{c r i t}<(1-k) s_{\min }$. The numerical values obtained show, regardless of the value of $C$, that $R_{H 1}$ leads to an $\mathbf{O P}$ characterized by very low values of $\varepsilon_{(\omega)}$ and $\varepsilon_{(s)}$. In order to increase the allowable range of $R$, the value of $k$ is increased for $R>R_{T}$. By doubling this quantity, a value for $R$ of $R_{H 2}$ is obtained which results from the nonsatisfaction of the condition applied to $\varepsilon_{(\omega)}$.

Taking the defined criteria into account, the controlled operation zone as determined by $C$ and $R$ is given in Figure 5.

The limiting curves LCL, LCH1 and LCH2 (fine lines) are deduced from the coordinates of the OPs corresponding to $R_{L}, R_{H 1}$ and $R_{H 2}$. The characteristics LCT (fine dashed line) and $\mathrm{LC}^{*}$ (bold line) corresponding respectively to $R_{T}$ and $R^{*}$ are also presented. The curves in Figure 6 give the variations of $\varepsilon_{(\omega)}$ and $e_{(\omega)}$ with $\omega_{\text {ref }}=\omega_{\min }$ (Fig. 6a) and of $\varepsilon_{(s)}$ and $\left.e_{(s}\right)$ with $s_{\text {ref }}=s_{\text {crit }}$ (Fig. 6b) in the controlled operation area determined by $C-R$. The solid

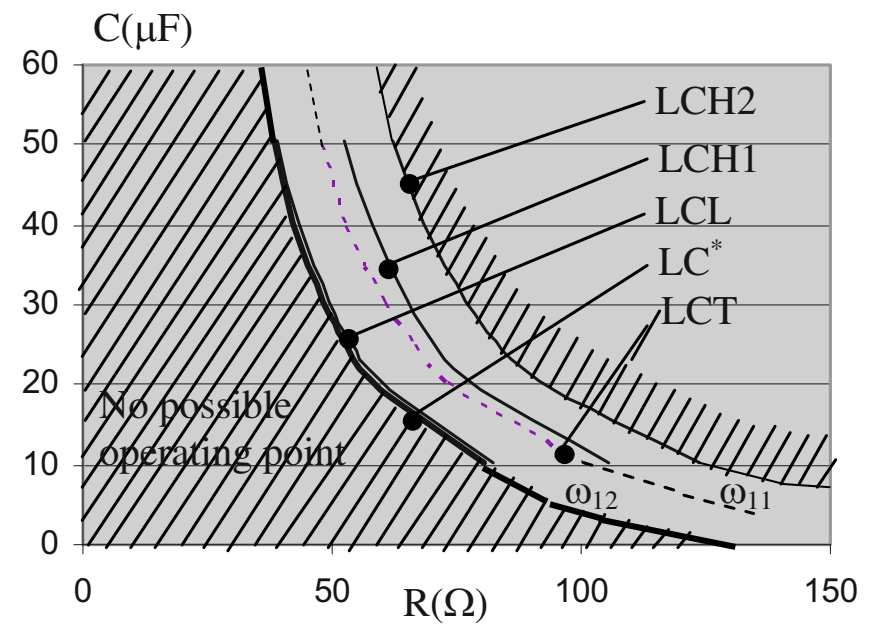

Fig. 5. Controlled operator area for combinations of $C$ and $R$.

curves correspond to the described estimations and the dashed curves correspond to the relative errors defined by equations (15).

It can be noticed that limiting the upper value of $R$ to $R_{H 1}$ leads to a sufficiently limited controlled operation area, approximately delimited by $R$ values of $R_{L}$ and $R_{T}$. It thus results that the $\mathbf{O P}$ would belong essentially to the characteristic $\omega_{12}(s)$.

Adapting the $k$ value by doubling it for $R>R_{T}$, is equivalent to nearly doubling the controlled operation zone, maintaining acceptable precision for the estimated values of $\omega$ and $s$ as is shown in the curves presented in Figure 6. These curves show that, in the SEIG controlled area, the estimated precisions are practically identical to the relative errors with an overestimation for low values of $R$ and an underestimation for high values. The minimal values of the estimated precisions or of the relative errors appear at values of $R$ close to $R_{T}$. To extend the allowed range of $R$ and $C$ it would be possible, in practice, to increase $R_{H 2}$, but such an increase would result in numerous disadvantages such as the expressions given by (27) becoming unreliable, and an $\mathbf{O P}$ close to a no-load condition for low values of $C$.

\section{Stability study}

The stability analysis will be carried out assuming that the SEIG load is only resistive. This resistance is varied starting from a stable steady state corresponding to a load $R_{0}$ with $C=C_{0}$. The problem consists of analyzing how the SEIG behaves during transients. Generally, as a first approach for this kind of study, it is assumed that the electrical time constants are much smaller than the mechanical one, which exists due to the polar moment of inertia $J$ of the whole rotational mechanical structure referred back to the SEIG shaft. That implies that the electrical transient has practically disappeared before the speed has a chance to vary significantly.

However, in this case, taking into account the uniqueness of the solution for the steady state of the system (11) 


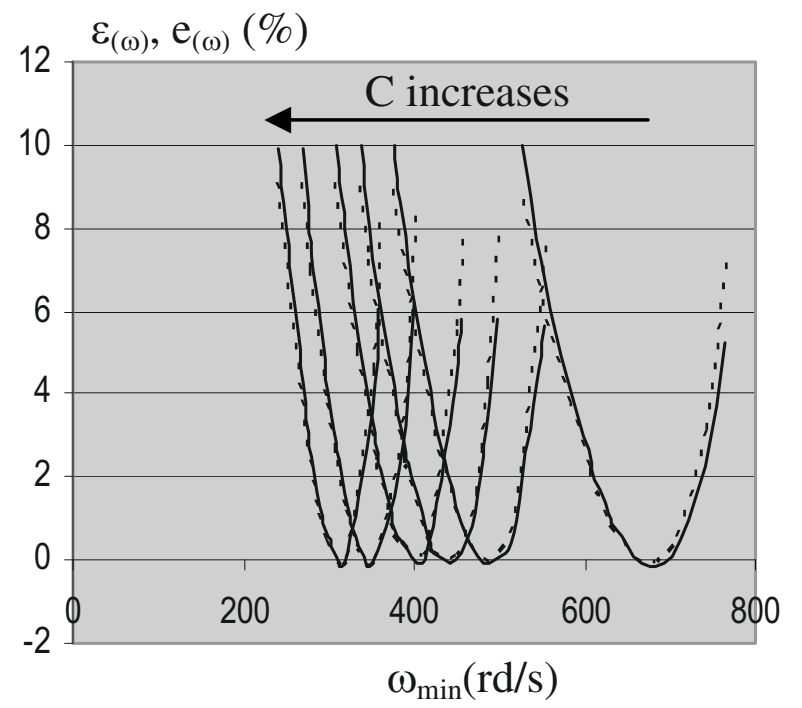

(a) $\varepsilon_{(\omega)}$ and $e_{(\omega)}$ versus $\omega_{\text {ref }}=\omega_{\min }$

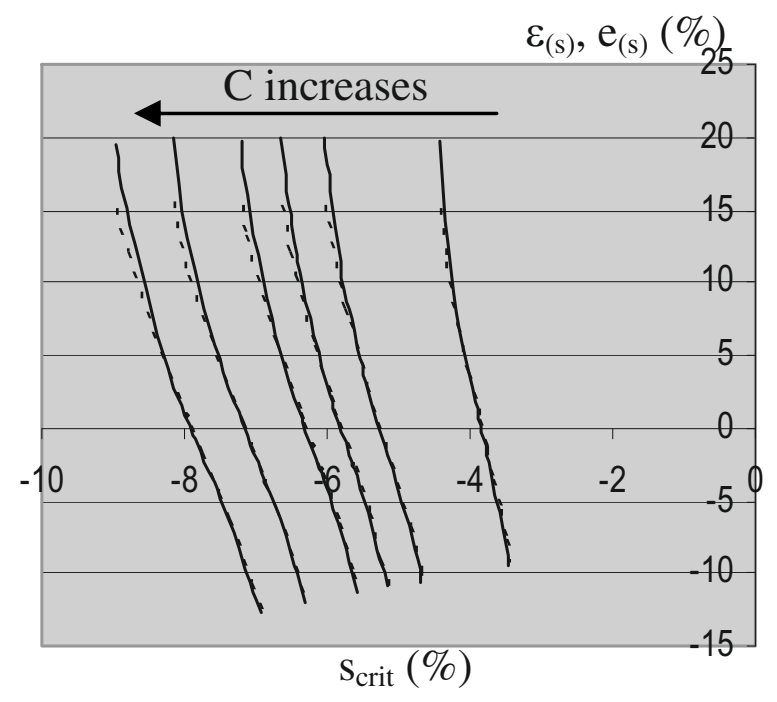

(b) $\varepsilon_{(\mathrm{s})}$ and $\mathrm{e}_{(\mathrm{s})}$ versus $\mathrm{s}_{\mathrm{ref}}=\mathrm{s}_{\mathrm{crit}}$

Fig. 6. Variations of the estimated precisions $\varepsilon(\omega), \varepsilon(s)(-)$ and of the relative errors $\left.e(\omega), e(s)(--)^{-}\right)$.

which defines a single rotor rotational speed for a given IM, $R$ and $C$, it is very probable that it will not be possible to dissociate the electrical and the mechanical transients. Consequently, any analysis of the SEIG behavior during these transients will be notably affected, and only a global modeling of the SEIG will be able to provide accurate information about the impact of changes in $R$.

\subsection{Global modeling of the Self-Excited Induction Generator}

The global model for the SEIG uses the space phasor formalism. It considers three-phase stator and rotor windings constituted of $n_{e}^{s}$ and $n_{e}^{r}$ effective turns for each phase. The stator $d^{s}$ and rotor $d^{r}$ spatial references are meant to be the same as the stator and rotor phase 1 axes. The self excitation of the SEIG is only possible if a remanent rotor flux density exists. To model this phenomenon, it is sufficient to consider three fictitious coils with $n_{e}^{a}$ effective turns each, distributed on the rotor just as the "real" winding. These coils are crossed by DC currents $i_{q}^{a}$ ( $q=1,2$ or 3 ) with a null sum. In this case, the space phasor $\underline{i}^{a}$ maintains a fixed modulus and position in the $d^{r}$ reference frame.

The analysis of the voltage equations is carried out in the rotor reference frame. Let us denote as $\underline{x}^{\text {s }}$ a stator space phasor $\underline{x}^{s}$ defined in the $d^{r}$ reference frame. One thus can obtain:

$$
\left.\begin{array}{l}
\underline{v}^{o s}=r^{s} \underline{i}^{o s}+\frac{d}{d t} \underline{\psi}^{o s}+j \omega^{\prime} \underline{\psi}^{o s} \\
\underline{v}^{r}=r^{r} \underline{\underline{i}}^{r}+\frac{d}{d t} \underline{\psi}^{r} \\
\underline{v}^{a}=r^{a} \underline{\underline{\underline{i}}}^{a}+\frac{d}{d t} \underline{\psi}^{a}
\end{array}\right\}
$$

where: $\omega^{\prime}=p \Omega^{\prime}, \underline{x}^{\circ}=\underline{x}^{s} \exp (-j \theta) \cdot \theta=\theta_{0}+\omega^{\prime} t$ defines the spatial angular gap between $d^{r}$ and $d^{s} . \underline{\psi}^{\circ}, \underline{\psi}^{r}$ and $\underline{\psi}^{a}$, which represent the fluxes linked by the stator, the rotor and the fictitious windings respectively, are expressed as:

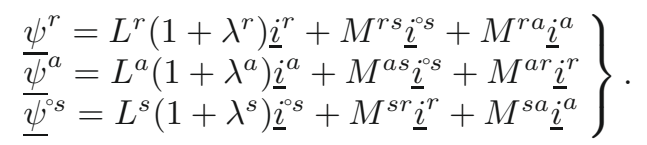

Introducing a constant $K$ tied to the IM geometric sizes, allows one to express the inductance coefficients:

- $L^{s}=K n_{e}^{s^{2}}, L^{r}=K n_{e}^{r^{2}}$ and $L^{a}=K n_{e}^{a^{2}}$ characterize the main cyclic inductances of the stator, the rotor and the fictitious windings,

- $M^{s r}=K n_{e}^{r} n_{e}^{s}, M^{s a}=K n_{e}^{s} n_{e}^{a}$ and $M^{r a}=K n_{e}^{r} n_{e}^{a}$ represent the mutual inductances between the stator, the rotor and the fictitious windings.

$-r^{s}, r^{r}$ and $r^{a}$ are the resistances of the stator, the rotor and the fictitious windings.

- $\lambda^{s}, \lambda^{r}$ et $\lambda^{a}$ are coefficients which characterize the leakage inductances.

Let us introduce the following quantities: $L^{\prime s}=L^{s}\left(1+\lambda^{s}\right)$, $L^{\prime r}=L^{r}\left(1+\lambda^{r}\right), L^{\prime a}=L^{a}\left(1+\lambda^{a}\right)$. By substituting the expression for $\psi^{\circ}$ into the stator voltage equation, as long as the time derivative of $\underline{i}^{a}$ is null and that the inductance coefficients are constants, one obtains:

$$
\begin{aligned}
\underline{v}^{o s}=\left[r^{s}+j \omega^{\prime} L^{\prime s}\right] \underline{i}^{o s}+L^{\prime s} \frac{d \underline{i}^{s}}{d t}+j \omega^{\prime} M^{s r} \underline{i}^{r} \\
+M^{s r} \frac{d \underline{i}^{r}}{d t}+j \omega^{\prime} M^{s a} \underline{i}^{a} .
\end{aligned}
$$


As $\underline{v}^{r}=0$, the rotor voltage equation makes it possible to express $\underline{\mathrm{i}}^{\text {os }}$ versus $\underline{i}^{r}$ and $\underline{i}^{a}$. This leads to:

$$
r^{r} \underline{i}^{r}+L^{\prime r} \frac{d \underline{i}^{r}}{d t}=-M^{s r} \frac{d \underline{i}^{\circ}}{d t} .
$$

In order to model the SEIG behavior, the following be also added to equations (33) and (34):

- the voltage equation which characterizes the impact of $R$ and $C$ in the rotor reference frame, as:

$$
-\underline{i}^{\circ s}=C \frac{d \underline{v}^{\circ} s}{d t}+\left(\frac{1}{R}+j \omega^{\prime} C\right) \underline{v}^{\circ}
$$

- the equation which represents the SEIG mechanical behavior, which will be assumed to be expressible as:

$$
\Gamma_{s}=\Gamma_{e}+\Gamma_{f}+J \frac{d \Omega^{\prime}}{d t}
$$

with $\Gamma_{s}=P_{w} / \Omega^{\prime}$ representing the torque which acts on the SEIG shaft by means of the blades, $\Gamma_{f}$ the SEIG friction and windage torque and $\Gamma_{e}$ the IM electromagnetic torque, as defined by the cross product:

$$
\Gamma_{e}=\frac{3}{2} \underline{\psi}^{\circ s} \times \underline{i}^{\circ s} .
$$

\subsection{Simulation results}

The IM considered in this section is the same as that which was the subject of the study in steady state. To exploit the equations which make it possible to model the SEIG behavior during transients, the variables $r^{r}, L^{r}$, $M^{s r}$ and $M^{s a}$ have to be characterized. It is assumed that the IM turn ratio at standstill is close to: $n_{e}^{s} / n_{e}^{r}=\sqrt{2}$. In these conditions, according to the numerical values of $r^{\prime r}, l^{\prime r}$ and $L^{s}$ given previously, one can deduce that: $l^{r}=$ $9 \mathrm{mH}, L^{r}=0.2 \mathrm{H}, r^{r}=1.8 \Omega, M^{s r}=\sqrt{L^{s} L^{r}}=0.283 \mathrm{H}$. To characterize $M^{s a}$ the value $0.1 \mathrm{H}$ is adopted. During the self excited step, the currents $i_{q}^{a}$ are such that: $\left|\underline{i}^{a}\right|=$ 0.1 A. When the SEIG is self-excited, the analysis of the steady state or of the transient states corresponding to changes in $R$, is performed assuming that the currents $i_{q}^{a}$ are null.

The different curves of Figure 7 display the modulus for certain values: $\Delta \underline{i}^{s}=\left|\underline{i}^{s}\right| / I_{(\text {rated })}^{s} \sqrt{2}, \Delta \underline{v}^{s}=$ $\left|\underline{v}^{s}\right| / V_{(\text {rated })}^{s} \sqrt{2}, \Delta \Gamma_{e}=-\Gamma_{e} / \Gamma_{e(\text { rated })}$ and $\Delta \Omega^{\prime}=$ $\Omega^{\prime} / \Omega_{(\text {rated })}$ with: $I_{(\text {rated })}^{s}=3.6 \mathrm{~A}, V_{(\text {rated })}^{s}=220 \mathrm{~V}$, $\Omega_{(\text {rated })}=157 \mathrm{rad} / \mathrm{s}, \Gamma_{e(\text { rated })}=11.5 \mathrm{Nm}$. The study is carried out assuming that the SEIG shaft is submitted to a constant $P_{w}$ equal to $-1700 \mathrm{~W}$.

A) Steady state. Starting from $t=0$ with zero speed, the SEIG evolves to reach the steady state corresponding to $R=R_{0}$ and $C=C_{0}$. The study is carried out for $J=0.4 \mathrm{kgm}^{2}, \Gamma_{f}=0 \mathrm{Nm}$. The steady state is reached when the space phasors $\underline{i}^{\circ}, \underline{i}^{r}$ and $\underline{v}^{\circ}$ possess constant moduli and turn at the same speed, $s \bar{\omega}$. This state, which appears at $t \cong 57 \mathrm{~s}$, is characterized by the following values: $s \omega=-29.60 \mathrm{rad} / \mathrm{s}, \omega^{\prime}=479.6 \mathrm{rad} / \mathrm{s}$ which lead to: $\omega=450 \mathrm{rad} / \mathrm{s}$ and $s=-6.57 \%$. These values are similar to those obtained under the same conditions during the steady state study. The moduli of the space phasors are: $\left|\underline{i}^{s}\right|=4.8 \mathrm{~A},\left|\underline{i}^{r}\right|=6.3 \mathrm{~A}$ and $\left|\underline{v}^{s}\right|=239.9 \mathrm{~V} . \Gamma_{e}$ is then equal to $-7.1 \mathrm{Nm}$.

B) Changes in $\mathbf{R}$. The starting point for this study corresponds to the previous steady state $\left(R=R_{0}, C=\right.$ $C_{0}, J=0.4 \mathrm{kgm}^{2}$ ) obtained for an instant $t=t^{*}$ just after $57 \mathrm{~s}$. At $t=t^{*}$ the value of $R$ is changed. Figure 5 shows that for $C=C_{0}$, the values of $R$ which lead to a controlled operating mode are between $53.5 \Omega$ and $83 \Omega$.

The curves shown in Figure 7 present the time variations of the related variables as $R$ takes on values of $55 \Omega$ and $80 \Omega$.

For these values, the SEIG tends to return towards a steady state after a relatively long transient. This transient is accompanied by large over-currents (in particular for $R=80 \Omega$ ) which last several seconds (close to $2 \mathrm{~s}$ for $R=55 \Omega$ ). These over-currents will likely trigger any electrical protection mechanisms present, disconnecting the SEIG from the load, and thus losing control of it.

These over-currents will also result in over-torques which can, if repeated, weaken the mechanical structure.

To limit these over-currents it would be possible to split up the increases or decreases in $R$ (progressive changes in $R$ ). However, this procedure will both make the command more complex, and will prolong large overvoltages at the load input, thus possibly degrading the load components.

C) Influence of $\mathbf{J}$. The previous curves show that the durations of the electrical transients are closely dependent on the mechanical time constant. To corroborate this result, it appears interesting to accompany the changes in $R$ at $t=t^{*}$ with changes in $J$.

The curves presented in Figure 8 give the results obtained when $R$ takes on the values $R=55 \Omega$ and $R=80 \Omega$, and $J$ takes on the value: $1.2 \mathrm{kgm}^{2}$. In both cases one can remark a disengagement of the SEIG along with a very large over-current and a very strong overtorque for $R=80 \Omega$.

These losses of control, as well as these large overvalues taken by some variables, are generated by the speed difference between the initial and final steady states that the SEIG must reduce. For $R=60 \Omega$, the steady state is characterized by $\Omega^{\prime}=239.8 \mathrm{rad} / \mathrm{s}$. The steady state speeds for $R=55 \Omega$ and $80 \Omega$ are equal respectively to: $\Omega^{\prime}=258.9 \mathrm{rad} / \mathrm{s}$ and $183.7 \mathrm{rad} / \mathrm{s}$ (these values are deduced from $(27))$. This leads differences with the initial speed of $-8 \%$ and $23.4 \%$. It is thus foreseeable that the problems will be all the more accentuated if $J$ is large.

\section{SEIG control during changes in $\mathbf{R}$}

To overcome the problems generated by the changes in $R$, the speed differences between the initial and final steady states must be limited. As $P_{w}$ does not intervene on the 

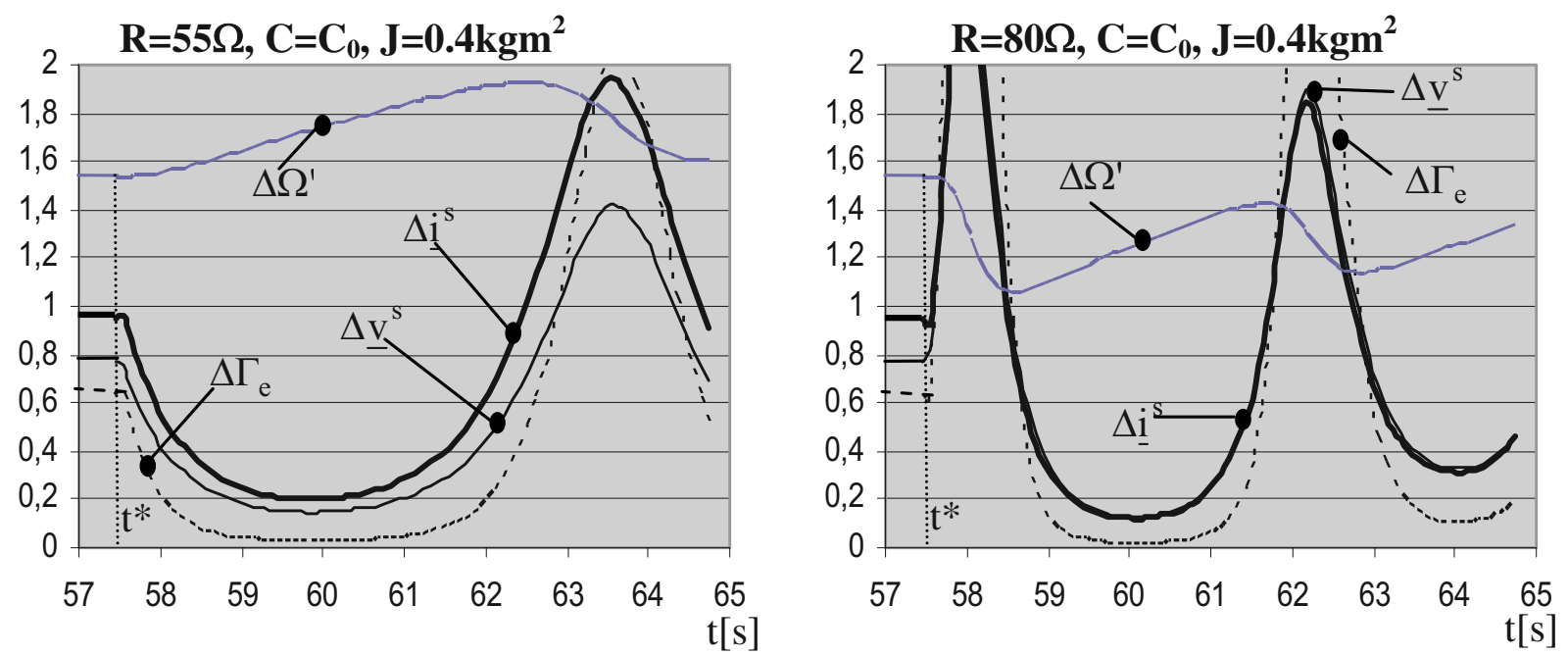

Fig. 7. Transients induced by changes in $R$ for $C=C_{0}$ and $J=0.4 \mathrm{kgm}^{2}$.
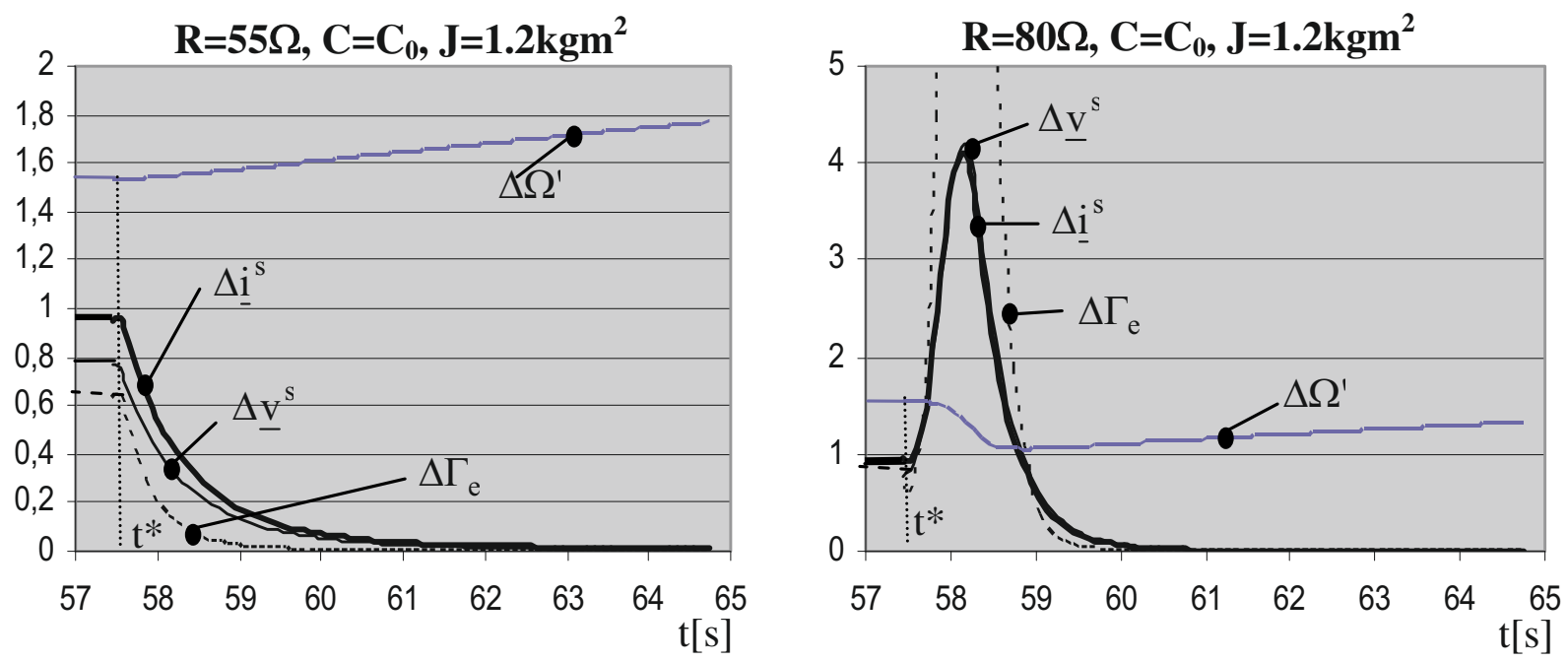

Fig. 8. Influence of $J$ on the SEIG behavior during transients tied to changes in $R$.

definition of these speeds, this analysis will be carried out assuming a constant $P_{w}$ equal to $-1700 \mathrm{~W}\left(P_{w}\right.$ only acts on the SEIG response time in accordance with equation (36)).

The examination of equations (27) shows that an increase of $R$ for given $C$, results in a decrease in $\omega$ and an increase in $s$. The increase in $s$ can only result from a decrease in the ratio $\Omega^{\prime} / \Omega$, and so the decrease in $\Omega^{\prime}$ must be greater than that of $\Omega$.

Considering again the first equation of system (27), it is possible to remark that a change in $R$, that keeps the $R C$ product equal, is equivalent, in the $C-R$ controlled operation regime, to keeping the angular frequency constant, and consequently also the frequency of the electrical variables delivered by the SEIG. The equation which gives the slip shows that $s$ increases with $R$. As previously, this increase can only result from a decrease in the ratio $\Omega^{\prime} / \Omega$. However, as $\Omega$ is constant, the decrease in $\Omega^{\prime}$, which is tied onto the changes in $s$, is considerably reduced compared to that necessary for a single increase in $R$. Consequently this procedure makes it possible to reduce the speed differences between the initial and final steady states, which should appreciably improve the SEIG behavior during the changes in $R$. To appreciate the impact of this speed difference reduction on the SEIG behavior, transient states for the two extreme values of $R$ $(55 \Omega$ et, $80 \Omega$ ) are presented in Figure 9 , for conditions when $J$ takes successively the values 0.4 and $1.2 \mathrm{kgm}^{2}$, and for constant RC product equal to $R_{0} C_{0}$. For the two extreme values of $R$ ( $55 \Omega$ et $80 \Omega$ ), C must take on values 
of $27.63 \mu \mathrm{F}$ and $19 \mu \mathrm{F}$ respectively. It is possible to check that these $R-C$ couples are indeed included in the $C-R$ controlled operation regime defined in Figure 5. The use of equations (27) leads, in the steady state, to speed differences with the initial speed of $-0.42 \%$ for $R=55 \Omega$ and $1.15 \%$ for $R=80 \Omega$. The curves shown in Figure 9 clearly demonstrate the beneficial effect of this procedure for the over-currents, the over-torques, as well for as the loss of control of the SEIG.

\section{Impact of this control law on the steady state}

It has been previously shown that operation while keeping the $R C$ product constant is equivalent, in steady state, to operation at a constant frequency. If this procedure makes it possible avoid loss of SEIG operation control, it is advisable to study the impact of this law on the possibility of voltage regulation. Moreover, it may be of interest to bring out the manner in which this control law acts on the quantities looked at above as $\omega_{e x}$ and $s_{e x}$. In the following, operational curves of the SEIG will be drawn towards the following considerations:

- to use the specific values $\omega_{e x}$ and $s_{e x}$ of $\omega$ and $s$ to define the different quantities (bold curves),

- to express these different quantities from $\omega_{\text {ref }}=\omega_{\text {min }}$ and $s_{\text {ref }}=s_{\text {crit }}$ (dashed curves).

\subsection{Impact on voltage control}

The impact on voltage control is considered at two different levels:

- The first one results from an examination of the simplified equation for $V^{s}$ given by (17). It reveals that for a given $\Gamma_{e}, V^{s}$ changes as $\sqrt{R \omega}$. Consequently, as $\omega$ and $R$ evolve in opposite direction, a change in $R$ alone will lead to a change in $V^{s}$ which will be lower than $\sqrt{R}$. It can also be deduced that a change in $R$ while keeping the $R C$ product constant, leads to a change in $V^{s}$ proportional to $\sqrt{R}$, assuming that $\omega$ is a constant as well. Consequently the depth of voltage regulation will be improved.

- The second point for consideration concerns the $C-R$ controlled operation regime as given in Figure 5 . To visualise the allowed variation in $R$ assuming operation at constant $C$, it suffices to draw a horizontal line at $C=C_{0}$. This shows that $R$ must be within the interval $\Delta R_{(C \text { const. })}=[53.5 \Omega ; 83 \Omega]$ as previously mentioned. By plotting the hyperbola $R C=R_{0} C_{0}$ it can be seen that by keeping $R C$ constant while varying $R$, a greater range of $R$ is permitted $\Delta R_{(R C \text { const. })}=[48 \Omega ; 115 \Omega]$.

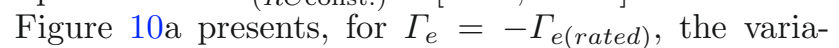
tions in $V^{s} / V_{(\text {rated })}^{s}$ with $R$ for the two ranges of $R$ as just defined, and using equation (13) to characterize $V^{s}$. In spite of an appreciable gap which affects the $V^{s}$ values according to they are calculated from $\omega_{\text {ref }}$ and $s_{\text {ref }}$ or $\omega_{e x}$ and $s_{e x}$, it appears that this constant frequency operation amplifies considerably the ease of voltage regulation. Concerning the gap as just evoked, it appears that this one keeps a constant value for operation at constant $R C$ while it increases with $R$ for operation at constant $C$. These particularities accentuate the benefits brought by this control law.

\subsection{Impact on frequency changes}

In order to better appreciate the meaning of this constant frequency operation, the curves given in Figure 10b present the variation in $\omega / \omega_{(\text {rated })}$ with $R$ for the two running modes: constant $C$ and constant $R C$ product. One can see that this natural frequency regulation (constant $R C$ product) gives excellent results over the interval $\Delta R_{(C \text { const. })}$ with a section (interval ab) where $\omega_{e x}$ is identical to $\omega_{\text {min }}$. Apart from this interval $\Delta R_{(C \text { const. })}$, the frequency performance deteriorate but keep nevertheless acceptable values. This particularity can be explained by considering the hyperbola $R C=R_{0} \mathrm{C}_{0}$ which, for a no negligible range in the changes in $R$, is relatively close to the LCL limit (see Fig. 5) for low values of $R$ and is close to the $\mathrm{LCH}_{2}$ limit for high values of $R$.

Let us note that this procedure, which leads to natural frequency control, induces small fluctuations in the frequency which are tied essentially to the non-linear character of the behaviour of the SEIG. In order to take this particularity into account, this procedure will be described as running at quasi constant frequency.

\section{Case of different load types}

- For a resistive load, $\varphi^{\prime s}$ as given by (8) can be expressed by $\operatorname{tg} \varphi^{\prime s}=R C \omega$. In these conditions, as $\omega$ can be considered as quasi constant for constant $R C$ product, it suffices to keep a constant $\operatorname{tg} \varphi^{\prime s}$ to obtain operation at quasiconstant voltage-frequency.

- When the nature of the load changes, as long as the characterization of the load as seen from the SEIG can always be done by means of the quantity $\operatorname{tg} \varphi^{\prime s}$, it seems probable that it suffices to satisfy a similar $\operatorname{rule}\left(\operatorname{tg} \varphi^{\prime s}\right.$ constant) to obtain operation at quasi constant $V^{s}$ and $f$. In this case the difficulty is deferred on any procedure which may make it possible to $\operatorname{keep} \operatorname{tg} \varphi^{\prime s}$ constant, as this quantity may be much more complex to control.

The aim is not to develop an analysis similar to that done for a resistive load but only to present the results obtained when considering voltage regulation if the suggested procedure is used. To do this, it is assumed, for example, that an inductance $L$ of constant value is connected at the terminals of the resistance $R$ in Figure 1. $\operatorname{tg} \varphi^{\prime s}$ is then given by:

$$
\operatorname{tg} \varphi^{\prime s}=R\left(L C \omega^{2}-1\right) / L \omega .
$$

Assuming that the voltage control is carried out by adapting the value of $R$, it appears, assuming that $\operatorname{keeping} \operatorname{tg} \varphi^{\prime s}$ constant leads to a quasi-constant value of $\omega$, that this 

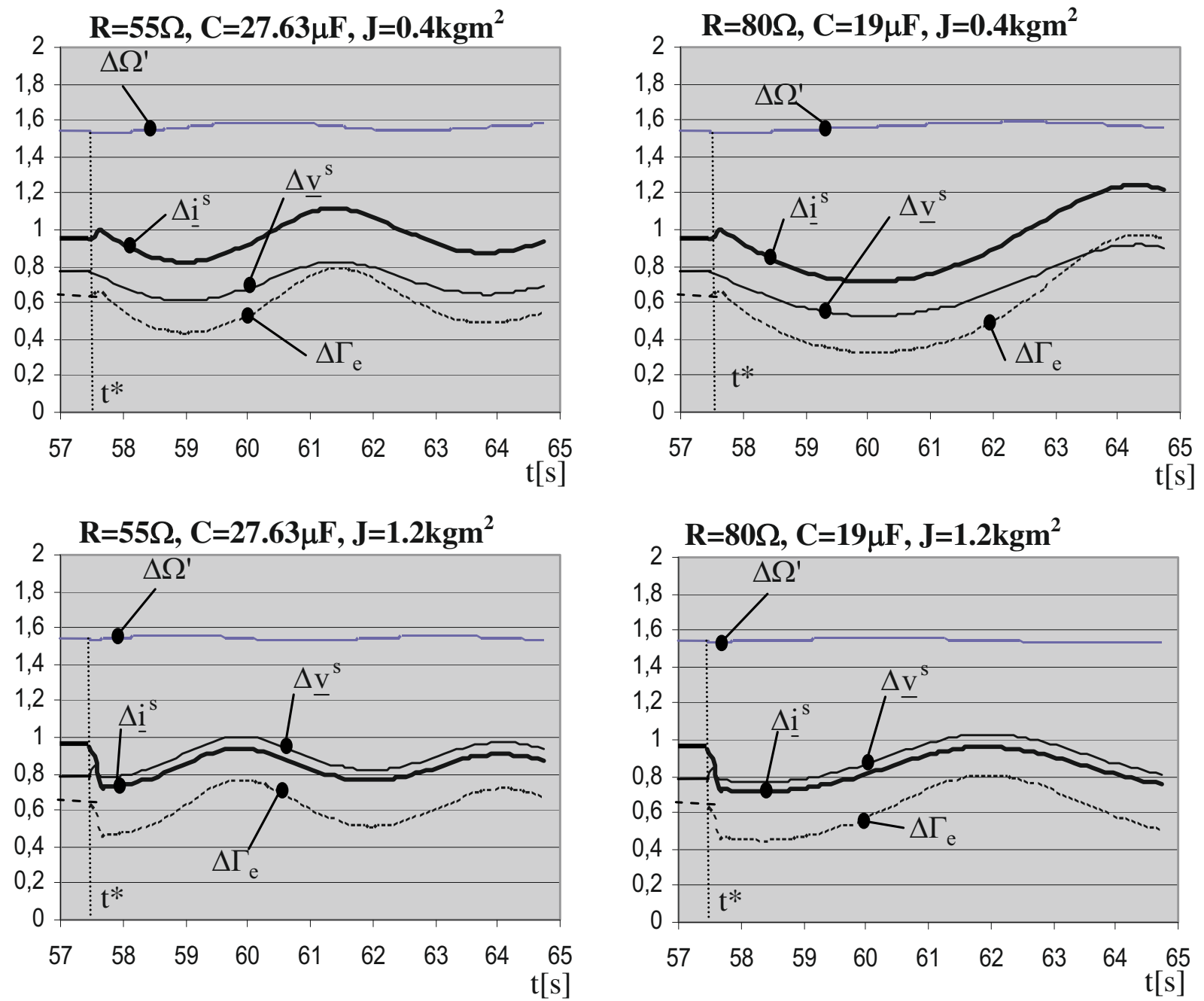

Fig. 9. Transients during the $R$ changes when the product $R C$ is constant and equal to $R_{0} C_{0}$.

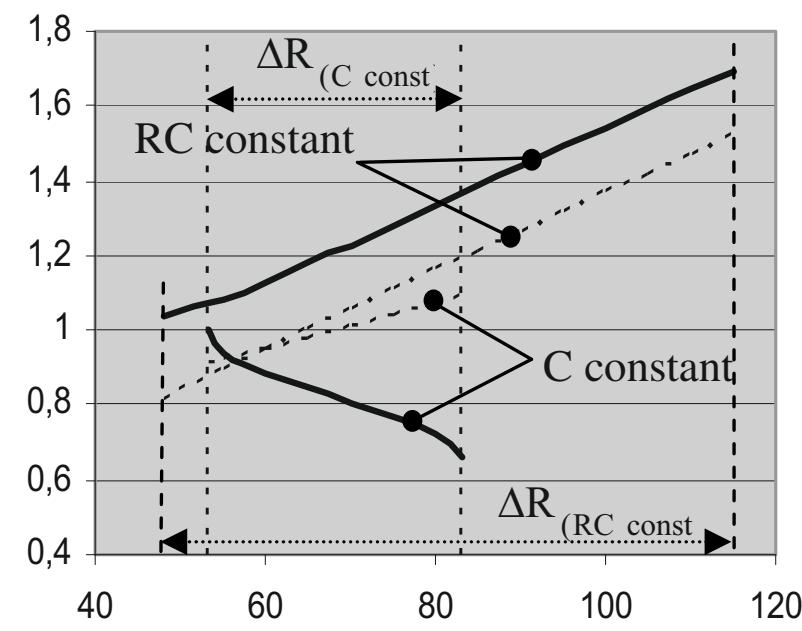

(a) Variations of $V^{s} / V_{(\text {rated })}^{s}$ with $R$.

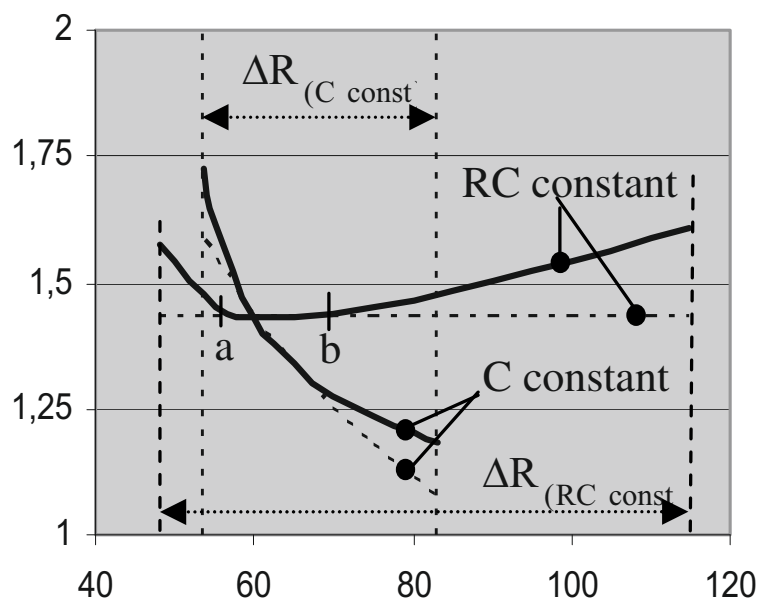

(b) Variations of $\omega / \omega_{(\text {rated })}$ with $R$.

Fig. 10. Variations of related variables for resistive load and $\Gamma_{e}=-\Gamma_{e(\text { rated })}$. 


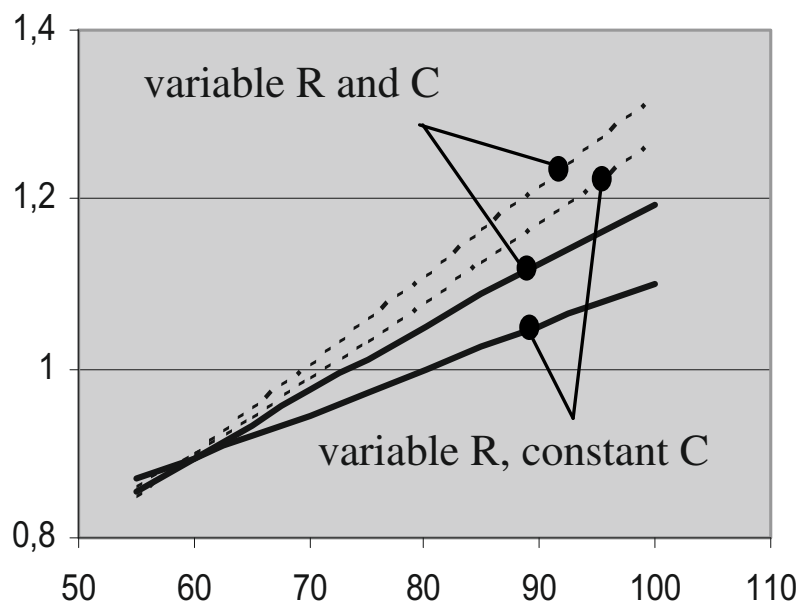

(a) Variations of $V^{s} / V_{(\text {rated })}^{s}$ with $R$.

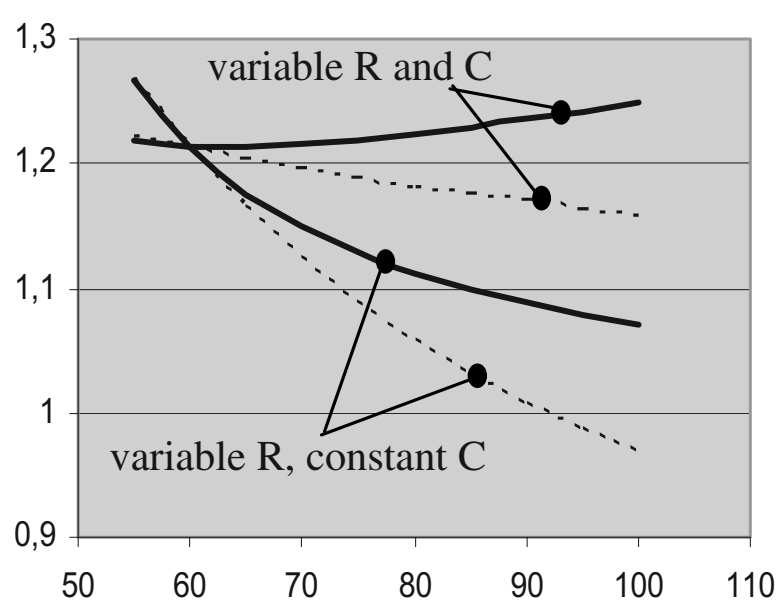

(b) Variations of $\omega / \omega_{(\text {rated })}$ with $R$.

Fig. 11. Variations of related variables for inductive load and $\Gamma_{e}=-\Gamma_{e(\text { rated })}$.

condition is satisfied when quantity $R\left(L C \omega^{2}-1\right)$ is kept constant.

When the parasitical terms are neglected, $\omega$ and $s$ are equal to $\omega_{0}^{\prime}$ and $s_{0}^{\prime}$, as defined by:

$$
\left.\begin{array}{l}
\omega=\omega_{0}^{\prime}=\sqrt{\left(L^{s}+L\right) / C L^{s} L} \\
s=s_{0}^{\prime}=-r^{\prime r} / R
\end{array}\right\} .
$$

For the IM considered and with $L=0.6 \mathrm{H}$, these equations show that in order to obtain $\omega \approx \omega_{(\text {rated })}$ and $s \approx-s_{(\text {rated })}$ it suffices to choose: $R=R_{0}=60 \Omega$, $C=C_{0}^{\prime}=42.2 \mu \mathrm{F}$. With the aim of keeping an angular frequency close to $\omega_{\text {(rated })}$, it is advisable, in order to keep $\operatorname{tg} \varphi^{\prime s}$ constant, to satisfy the equality: $R\left(L C \omega_{(\text {rated })}^{2}-1\right)=$ $R_{0}\left(L C_{0}^{\prime} \omega_{(\text {rated })}^{2}-1\right)$. This leads to the following relationship between $C$ and $R$ :

$$
C=\left[R+R_{0}\left(L C_{0}^{\prime} \omega_{(\text {rated })}^{2}-1\right)\right] / R L \omega_{(\text {rated })}^{2} .
$$

The relationship between $V^{s}$ and $\Gamma_{e}$ can be written as:

$$
V^{s}=R \frac{L}{L^{s}} \sqrt{\frac{\Gamma_{e} \omega}{3 p} \frac{r^{\prime r 2}+s^{2} L^{s 2}\left(1+\lambda^{r}\right)^{2} \omega^{2}}{\left[R^{2}\left(L C \omega^{2}-1\right)^{2}+L^{2} \omega^{2}\right] s r^{\prime r}}} .
$$

As previously, it is possible to characterize $\omega$ and $s$ by setting them equal to $\omega_{\text {ref }}=\omega_{\text {min }}$ and $s_{\text {ref }}=s_{\text {crit }}$. Following the same approach that was used during the study with a resistive load and when still using the constants given by (10), one can in this case obtain:

$$
\left.\begin{array}{l}
s_{\text {ref }}=s_{\text {crit }}=\frac{L L^{s 2} r^{\prime r}}{2\left(R A^{\prime}-L E^{\prime \prime}\right)}\left(1+\sqrt{1-\frac{4 R C D^{\prime}\left(R A^{\prime}-L E^{\prime \prime}\right)}{L L^{s 4} r^{\prime} r^{2}}}\right) \\
\omega_{r e f}^{2}=\omega_{\text {min }}^{2}=-\frac{r^{s} E^{\prime} s_{\min }^{2}+L^{s 2} r^{\prime r} R s_{\min }-L D^{\prime \prime}}{L s_{\min }\left(A^{\prime \prime} s_{\min }-B\right)}
\end{array}\right\}
$$

where:

$$
\begin{aligned}
s_{\text {min }} & =\frac{A^{\prime \prime} L D^{\prime \prime}}{r^{s} E^{\prime} B+L^{s 2} r^{\prime r} R A^{\prime \prime}} \\
& \times\left\{1+\sqrt{1-\frac{B}{A^{\prime \prime 2} L D^{\prime \prime}}\left(r^{s} E^{\prime} B+L^{s 2} r^{\prime r} R A^{\prime \prime}\right)} .\right.
\end{aligned}
$$

These equations show that simply inserting the element $L$ leads to $\omega_{\text {min }}$ and $s_{\text {crit }}$ relationships which are much more complex.

- The curves of Figure 11a present the variations of $V^{s} / V_{\text {(rated) }}^{s}$ with $R$ deduced from (41) for a given $\Gamma_{e}$ equal to $-\Gamma_{e(\text { rated })}$ considering the following two strategies: [variable $R$; constant $C$ ] and [variable $R$ and $C$ ]. Let us note that these curves are drawn without consideration as to the potential limitations imposed by the controlled operation regime, as this complication would be to tedious to present in this paper. It appears again that the suggested procedure increases the ease of voltage regulation.

- The curves of Figure $11 \mathrm{~b}$ give the variations of $\omega / \omega_{(\text {rated })}$ with $R$ for the two previously described strategies. Again it is possible to appreciate the positive impact brought by the additional degree of freedom that this suggested control law introduces, in this case (it suffices to choose a value other than $\omega_{(\text {rated })}$ in equation (40) in order to change the variations of the frequency with $R$ ).

\section{Conclusion}

The analysis presented in this paper is based on a singlephase equivalent circuit definition using the notion of an induced voltage source in order to characterize SEIG operation. It has made it possible to overcome the inherent difficulties related to the inverse nature of the problem, when considering a resistive load in steady state. 
However, it has been necessary to define an operating regime within which the SEIG behavior is to be controlled. The other advantage of this operational regime is the possibility to characterize the angular frequency and the slip within this region by analytical expressions, thus taking into account all the parameters which characterize the IM. From these expressions a control strategy has been proposed. This strategy makes it possible, to avoid the over-values taken by some variables and to avoid the disengagement of the SEIG during the state changes imposed by the load resistance variations necessary to control the voltage appearing at the inputs.

This aspect is very interesting because these phenomena are often prejudicial with the development of such structures at remote sites. The SEIG behavior thus obtained can be considered as operation at quasi constant voltage-frequency without a frequency control loop. This command law also makes it possible to increase the ease of voltage regulation. Finally, it has been shown that this regulation strategy could be used in the case of loads of different types as long as the relationship between $\mathrm{C}$ and the load components is adapted.

\section{Abbreviations}

$\begin{array}{ll}\text { IM } & \text { Induction Machine } \\ \text { SEIG } & \text { Self-Excited Induction Generator } \\ \text { OP } & \text { Opperating } \underline{\text { Point }}\end{array}$

\section{References}

1. S. Heir, Wind energy conversion systems (John Wiley \& Sons, 1998), ISBN: 047197143 X

2. T.S. Bhatti, A.A.F. Al-Amedi, N.K. Bansal, Energy Convers. Mgmt. 38, 9 (1997)
3. E.S. Ganidou, A.G. Bahirtzis, P.S. Dokopoulos, IEEE Trans. Energy Convers. 7, 3 (1992)

4. A. Ilinca, J.L. Chaumel, G. Thibault, in Proceedings of the International Conference on Wind energy and remote regions, Magdalen islands, Canada, 2005, p. 294

5. R.C. Bansal, IEEE Trans. Energy Convers. 20, 2 (2005)

6. S. Rajakaruna, R. Bonert, IEEE Trans. Energy Convers. 8, 4 (1993)

7. R.J. Harrington, F.M.M. Bassiouny, IEEE Trans. Energy Convers. 13, 3 (1998)

8. L.C.A. Lopes, R.G. Almeida, IEEE Trans. Energy Convers. 21, 2 (2006)

9. M.S. Miranda, R.O.G. Lyra, S.R. Sylva, IEEE Trans. Energy Convers. 14, 4 (1999)

10. M.G. Simoes, B.K. Bimal, R.J. Spiegel, IEEE Trans. Power Electronics 12, 1 (1997)

11. J.F. Maissan, in Proceedings of the International Conference on Wind energy and remote regions, Magdalen islands, Canada, 2005, p. 194

12. A.L. Alolah, M.A. Alkanhal, IEEE Trans. Energy Convers. 15, $1(2000)$

13. H. Roisse, G. Peset, V. Autier, J.F. Brudny, in Proceedings of the 15th International Conference on Electrical Machines ICEM 2002, Bruges, 2002, p. 452

14. H. Roisse, G. Peset, V. Autier, J.F. Brudny, in Proceedings of the International Conference on Renewable Energy and Power Quality ICREPQ'2003, Vigo, 2003, p. 426

15. J.F. Brudny, H. Roisse, G. Peset, in Proceedings of the International Conference on Wind energy and remote regions Magdalen islands, Canada, 2005, p. 13 